\title{
Nonlinear dynamics of two-layer channel flow with soluble surfactant below or above the critical micelle concentration
}

\author{
A. Kalogirou ${ }^{1} \dagger$ and M. G. Blyth ${ }^{2}$ \\ ${ }^{1}$ School of Mathematical Sciences, University of Nottingham, University Park, Nottingham \\ NG7 2RD, United Kingdom \\ ${ }^{2}$ School of Mathematics, University of East Anglia, Norwich Research Park, Norwich NR4 \\ 7TJ, United Kingdom
}

(Received $\mathrm{xx}$; revised $\mathrm{xx}$; accepted $\mathrm{xx}$ )

The nonlinear stability of an inertialess two-layer surfactant-laden Couette flow is considered. The two fluids are immiscible and have different thicknesses, viscosities, and densities. One of the fluids is contaminated with a soluble surfactant whose concentration may be above the critical micelle concentration (CMC), in which case micelles are formed in the bulk of the fluid. A surfactant kinetic model is adopted that includes the adsorption and desorption of molecules to and from the interface, and the formation and breakup of micelles in the bulk. The lubrication approximation is applied and a strongly nonlinear system of equations is derived for the evolution of the interface and surfactant concentration at the interface, as well as the vertically-averaged monomer and micelle concentrations in the bulk (as a result of fast vertical diffusion). The primary aim of this study is to determine the influence of surfactant solubility on the nonlinear dynamics. The nonlinear lubrication model is solved numerically in periodic domains and saturated travelling waves are obtained at large times. It is found that a sufficiently soluble surfactant can either destabilise or stabilise the interface depending on certain fluid properties. The stability behaviour of the system depends crucially on the values of the fluid viscosity ratio $m$ and thickness ratio $n$ in reference to the boundary $m=n^{2}$. If the surfactant exists at large concentrations that exceed the $\mathrm{CMC}$, then long waves are stable at large times, unless density stratification effects overcome the stabilising influence of micelles. Travelling wave bifurcation branches are also calculated and the impact of various parameters (such as the domain length or fluid thickness ratio) on the wave shapes, amplitudes and speeds is examined. The mechanism responsible for interfacial (in)stability is explained in terms of the phase difference between the interface deformation and concentration waves, which is shifted according to the sign of the crucial factor $\left(m-n^{2}\right)$ and the strength of the surfactant solubility.

\section{Introduction}

Surfactants are surface active agents that are known to affect the surface tension of fluids (Chang \& Franses 1995). Experimental measurements of equilibrium surface tension have demonstrated that the surface tension is reduced in response to the concentration of surfactant in the bulk of the fluid (Song et al. 2006). The surface tension behaviour is also affected by the process of micellisation, which takes place when the bulk concentration of monomers reaches a critical value; this value is defined as the critical micelle concentration $(\mathrm{CMC})$ and also signifies the stage where the surface tension is decoupled from further

$\dagger$ Email address for correspondence: Anna.Kalogirou@nottingham.ac.uk 
variations in surfactant concentration and attains a uniform value. The equilibrium and dynamic surface tension behaviour of surfactants is important in a range of processes and applications, as it can influence foam fabrication and stability (Petkova et al. 2012), affect wettability in coatings (Weinstein \& Ruschak 2004) (e.g. in photographic applications (Maisch et al. 2019), and control film deformation (Afsar-Siddiqui et al. 2003). The latter property of surfactants is also significant in multilayer flows, where surfactants can be used to manipulate deformations at fluid-fluid interfaces - this is possible due to the dynamic variation of surface tension and resulting Marangoni forces (e.g. Pozrikidis 2004). The effect of surfactants on linear and nonlinear stability of multilayer flow has been investigated extensively, both theoretically and numerically; a summary of the most prominent studies, in the context of surfactant-free or surfactant-laden flows, is given next.

In the absence of surfactant, Yih (1967) considered a shear flow of two immiscible viscous fluids in a channel and investigated the stability properties of the system when the interface is subject to large-wavelength perturbations. He found that instability is manifested as long as the fluid viscosities are different and the Reynolds number is nonzero (assuming the fluids have equal densities). Hooper (1985) studied a semi-infinite shear flow and reported that Yih's long-wave instability emerges only when the thin fluid is the more viscous. This result was confirmed by Renardy (1985) who solved the Orr-Sommerfeld equations for arbitrary wavelengths. In the case of a two-layer flow in an inclined channel, a linear stability analysis was performed by Tilley et al. (1994a) for perturbations of either large or arbitrary wavelength. The authors also examined the competition between the different mechanisms (e.g. due to density or viscosity stratification) for interfacial instability that arise in such flows.

In all the aforementioned linear stability studies, the interface was devoid of surfactant. The influence of insoluble surfactants on the stability properties of a two-layer shear flow was investigated by Frenkel \& Halpern (2002); Halpern \& Frenkel (2003) and Blyth \& Pozrikidis (2004). It was found that the presence of surfactants gives rise to destabilising Marangoni stresses and induces interfacial instability even under conditions supporting a stable clean interface, namely when the fluids' viscosities are equal or when inertial effects are negligible. The combined effects of gravity and Marangoni forces on flow stability were examined in Frenkel et al. (2019a b $)$. More recently, a number of studies considered the surfactant to be soluble in one or both fluids and analysed the impact of surfactant solubility on the linear stability of two-layer channel flows. Picardo et al. (2016) investigated the effect of Marangoni forces on the stability and also studied the effect of inertia on the solutal Marangoni instability. Their analysis was, however, based on the simplifying assumption of instantaneous adsorption/desorption of surfactant from one phase to the other and neglected the interfacial transport of surfactant. The dynamic transport of surfactant at the interface due to adsorption was included in the work of Kalogirou \& Blyth (2019), who formulated a model that takes into account the possibility of surfactant concentrations exceeding the CMC. The authors analysed the linear stability properties of the model both numerically for disturbances of arbitrary wavelength and analytically using long-wave approximations, for a range of fluid properties.

The identified linear instabilities were followed into the nonlinear regime in several studies. A nonlinear evolution equation describing the propagation of long finiteamplitude waves at the interface between two viscous fluids (free from surfactants) was obtained and solved numerically by Ooms et al. (1985). Hooper \& Grimshaw (1985) and Renardy (1989) both derived a Kuramoto-Sivashinsky equation for the weakly nonlinear evolution of the interface, and found steady state and travelling wave solutions. Tilley et al. (1994b) investigated the nonlinear stability of a two-layer flow in an inclined channel 
and derived a strongly nonlinear equation for the evolution of long waves at the interface; their evolution equation reduces to a Kuramoto-Sivashinsky-type equation in the weakly nonlinear limit. When insoluble surfactants are added at the interface, a similar long-wave analysis was followed by Blyth \& Pozrikidis (2004) and a set of two partial differential equations were obtained, coupling the evolution of the interface and its local surfactant concentration. That work was later extended by Frenkel \& Halpern (2017) to include the effect of density stratification in the system and to examine the interacting effects of gravity and Marangoni forces. Furthermore, the impact of insoluble surfactant on the stability of an interface between a thin film and a much thicker fluid has been analysed in the studies of Bassom et al. (2010); Kalogirou et al. (2012); Kalogirou \& Papageorgiou (2016); Kalogirou (2018) through analysis and numerical computations of a system of evolution equations that includes a nonlocal contribution from the thicker fluid.

In this work, we consider a two-layer surfactant-laden flow in a channel, subject to background shear due to the motion of the upper channel wall (Couette flow) as well as solutal Marangoni effects. The surfactant exists in three phases: as interfacial monomers, monomers in the bulk of one of the fluids, and spherical micellar aggregates (if the bulk concentration exceeds the CMC). Both fluids are considered to be Newtonian, thereby ignoring the possibility of the surfactant mass in the bulk growing well beyond the CMC, or equivalently, not allowing the micelle concentration to become very large. When this happens, the spherical micelles transition to cylindrical "wormlike" micelles that behave like polymers and start affecting the rheology of the fluid (Berret 2006); such cases are not considered in this study. A complete mathematical model is presented describing the dynamics of the flow and the transport of surfactant at the interface and in the bulk fluid (for both monomers and micelles). We perform a lubrication analysis that reduces the system to a simplified set of equations for the evolution of long waves at the interface and the variations of the respective surfactant concentrations. The effects of inertia are negligible in the leading-order dynamics and therefore the final evolution equations correspond to an inertialess flow. The obtained long-wave model is strongly nonlinear and reduces to that of Tilley et al. (1994b) for zero Re when surfactant is absent, or to the model derived in Blyth \& Pozrikidis (2004), Frenkel \& Halpern (2017) if the surfactant is considered to be insoluble. We carry out time-dependent numerical simulations of the reduced model for several cases motivated from predictions of the linear stability analysis of Kalogirou \& Blyth (2019). We also analyse the effect of surfactant solubility and other parameters on the interfacial travelling waves. Finally, the mechanism for interfacial instability is explained based on the phase shift between interfacial deformation and concentration (Wei 2005).

The paper is organised as follows. Section 2 presents the mathematical formulation of the problem considered in this study. The governing equations and boundary conditions are first given $(\$ 2.1)$ and the asymptotic model is then derived based on the lubrication approximation (2.2). In section 3 the numerical methods and results are presented, including linear growth rates $(\$ 3.2)$, nonlinear travelling wave solutions obtained from time-dependent simulations of the model for a range of parameter sets (\$3.3), and travelling wave branches constructed directly using continuation techniques (\$3.4). A brief explanation of the (in)stability mechanism is given in section 4 . The main conclusions are discussed in section 5 .

\section{Mathematical formulation}

We consider the evolution of the two-fluid system in a channel of uniform height $d$, as shown in figure 1 . The flow in each fluid region will be denoted using subscripts 1 and 2 for 


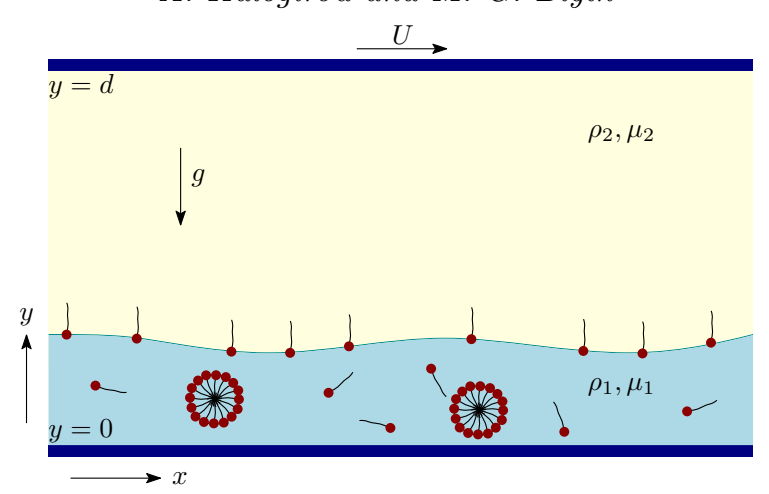

FiguRE 1. Sketch of the problem statement, including the coordinate system, geometrical specifications of the channel and physical properties of the fluids. The size of the surfactant molecules shown in the lower fluid is not to scale.

the bottom and top fluids, respectively. The two fluids are immiscible and incompressible and have in general different viscosities $\mu_{1}, \mu_{2}$ and densities $\rho_{1}, \rho_{2}$. The system is subject to the action of gravity $g$ and the shearing motion of the upper channel wall with speed $U$. We define a two-dimensional Cartesian coordinate system $(x, y)$, with horizontal coordinate $x$, vertical coordinate $y$ and time $t$. The bottom fluid then extends from $y=0$ up to the interface at $y=h(x, t)$, while the top fluid occupies the region $h(x, t) \leqslant y \leqslant d$. We also define the $2 \mathrm{D}$ gradient by $\nabla=\left(\partial_{x}, \partial_{y}\right)$ and in each fluid layer $i=1,2$ we introduce the pressure $p_{i}(x, y, t)$ and velocity field $\boldsymbol{u}_{i}(x, y, t)$, with horizontal velocity $u_{i}(x, y, t)$ and vertical velocity $v_{i}(x, y, t)$. The bottom fluid is laden with surfactant that can also get adsorbed at the interface and can potentially reach high concentrations exceeding the critical micelle concentration, in which case micelles are formed in the bulk. The interfacial, bulk and micellar species admit concentrations $\Gamma(x, t), C(x, y, t)$ and $M(x, y, t)$, and diffusivities $\mathscr{D}_{s}, \mathscr{D}_{b}$ and $\mathscr{D}_{m}$, respectively. The interfacial surfactant concentration $\Gamma$ alters the surface tension $\gamma$ according to the Langmuir equation of state (Chang \& Franses 1995). Each micelle is assumed to comprise $N$ monomers; the exchange between monomers and micelles during the micellisation process is described by the flux $J_{m}=k_{b} C^{N}-k_{m} M$, with micelle formation and breakup rates $k_{m}, k_{b}$, respectively. Finally, the sorption kinetics describing the exchange of surfactant molecules between the bulk and the interface are realised through the flux $J_{b}=k_{a} C\left(1-\Gamma / \Gamma_{\infty}\right)-k_{d} \Gamma$, with adsorption/desorption kinetic rates $k_{a}, k_{d}$. Here, $\Gamma_{\infty}$ denotes the maximum packing concentration at the interface, which when reached, leads to suspension of the adsorption process.

The system outlined above can be described mathematically by writing appropriate equations for the conservation of mass and momentum, as well as advection-diffusion equations for each surfactant species (Kalogirou \& Blyth 2019). The problem is written in non-dimensional form by rescaling lengths using the channel height $d$, velocities using the speed of the upper wall $U$, pressures using $\mu_{1} U / d$, time using $d / U$, surface tension using the clean reference value $\gamma_{0}$, interfacial surfactant concentration using the maximum packing concentration $\Gamma_{\infty}$, bulk monomer concentration using the critical micelle concentration $C_{C M C}=\left(k_{m} / N k_{b}\right)^{1 /(N-1)}$ (Breward \& Howell 2004), and micelle concentration using $C_{C M C} / N$. The total mass of surfactant is rescaled by $\mathcal{L} \Gamma_{\infty}$, where $\mathcal{L}$ is the length of an (arbitrary) horizontal domain. Typical values of all the physical parameters are provided in table 1 . The pertinent non-dimensional parameters are defined in table 2 together with some typical values based on the physical parameters in table 1 . 
Parameter [units]

Fluid viscosity $\left[\mathrm{N} \cdot \mathrm{s} / \mathrm{m}^{2}\right]$

Fluid density $\left[\mathrm{kg} / \mathrm{m}^{3}\right]$

Wall speed $[\mathrm{m} / \mathrm{s}]$

Channel height $[\mathrm{m}]$

Interfacial tension $[\mathrm{N} / \mathrm{m}]$

Gravity $\left[\mathrm{m} / \mathrm{s}^{2}\right]$

Ideal gas constant $[\mathrm{N} \cdot \mathrm{m} / \mathrm{K} \cdot \mathrm{mol}]$

Absolute temperature $[\mathrm{K}]$

Packing concentration $\left[\mathrm{mol} / \mathrm{m}^{2}\right]$

Critical micelle concentration $\left[\mathrm{mol} / \mathrm{m}^{3}\right]$

Surface diffusion $\left[\mathrm{m}^{2} / \mathrm{s}\right]$

Bulk diffusion $\left[\mathrm{m}^{2} / \mathrm{s}\right]$

Micelle diffusion $\left[\mathrm{m}^{2} / \mathrm{s}\right]$

Desorption rate $[1 / \mathrm{s}]$

Sorption rate ratio $[\mathrm{m}]$

Micelle formation rate $[1 / \mathrm{s}]$

Micelle size

$\begin{array}{lll}\text { Symbol } & \text { Values } & \text { References } \\ \mu_{1,2} & 1-3 \times 10^{-3} & \mathrm{SH} \\ \rho_{1,2} & 850-1200 & \mathrm{SH}, \mathrm{BA} \\ U & 0.05-5 & \mathrm{BA}, \mathrm{PK} \\ d & 10^{-6}-10^{-2} & - \\ \gamma_{0} & 0.035-0.072 & \mathrm{SH} \\ g & 9.81 & - \\ \mathcal{R} & 8.314 & \mathrm{SO}, \mathrm{MO} \\ \mathcal{T} & 298.15 & \mathrm{SO}, \mathrm{MO} \\ \Gamma_{\infty} & 1-10 \times 10^{-6} & \mathrm{SO}, \mathrm{SH}, \mathrm{CL}, \mathrm{CF}, \mathrm{PH} \\ C_{c m c} & 10^{-2}-10^{0} & \mathrm{SO}, \mathrm{MO}, \mathrm{CL} \\ \mathscr{D}_{s} & 10^{-10}-10^{-8} & \mathrm{SH}, \mathrm{MO}, \mathrm{PK} \\ \mathscr{D}_{b} & 10^{-9}-10^{-7} & \mathrm{MO} \\ \mathscr{D}_{m} & 10^{-9}-10^{-7} & \mathrm{MO} \\ k_{d} & 10^{-2}-10^{2} & \mathrm{SH} \\ k_{a} / k_{d} & 10^{-9}-10^{1} & \mathrm{CF} \\ k_{m} & 10-75 & \mathrm{MO}, \mathrm{DA} \\ N & 5-100 & \mathrm{ED}, \mathrm{MA}, \mathrm{DA}\end{array}$

TABLE 1. Typical values of the physical parameters describing the geometrical specifications, properties of the fluids and the surfactants. Key to references: SH: Shen et al. (2002), BA: Barthelet et al. (1995), PK: Pereira \& Kalliadasis (2008), SO: Song et al. (2006), MO: Morgan et al. (2012), CL: Chen \& Lee (2000), CF: Chang \& Franses (1995), PH: Phan et al. (2005), DA: Danov et al. (1996), ED: Edmonstone et al. (2006), MA: Mavromoustaki et al. (2012a).

\subsection{Governing equations}

The dynamics within the two fluid layers $i=1,2$ are governed by the following nondimensional equations

$$
\begin{aligned}
\nabla \cdot \boldsymbol{u}_{i} & =0, \\
R e\left(\boldsymbol{u}_{i t}+\boldsymbol{u}_{i} \cdot \nabla \boldsymbol{u}_{i}\right) & =-\frac{1}{r_{i}} \nabla p_{i}+\frac{m_{i}}{r_{i}} \nabla^{2} \boldsymbol{u}_{i}-\frac{B o}{C a} \hat{\boldsymbol{y}}, \\
C_{t}+\boldsymbol{u}_{1} \cdot \nabla C & =\frac{1}{P e_{b}} \nabla^{2} C-J_{m}, \\
M_{t}+\boldsymbol{u}_{1} \cdot \nabla M & =\frac{1}{P e_{m}} \nabla^{2} M+J_{m},
\end{aligned}
$$




$\begin{array}{clll}\text { Parameter } & \text { Definition } & \text { Description } & \text { Value range } \\ m & \mu_{2} / \mu_{1} & \text { Viscosity ratio } & 0.3-3 \\ r & \rho_{2} / \rho_{1} & \text { Density ratio } & 0.7-1.4 \\ R e & \rho_{1} U d / \mu_{1} & \text { Reynolds number } & 10^{-2}-10^{5} \\ C a & \mu_{1} U / \gamma_{0} & \text { Capillary number } & 10^{-3}-10^{-1} \\ B o & d^{2} \rho_{1} g / \gamma_{0} & \text { Bond number } & 10^{-7}-10^{1} \\ M a & \mathcal{R T} \Gamma_{\infty} / \mu_{1} U & \text { Marangoni number } & 10^{-1}-10^{2} \\ P e_{s} & U d / \mathscr{D}_{s} & \text { Surface Péclet number } & 10^{1}-10^{8} \\ P e_{b} & U d / \mathscr{D}_{b} & \text { Bulk Péclet number } & 10^{0}-10^{7} \\ P e_{m} & U d / \mathscr{D}_{m} & \text { Micelle Péclet number } & 10^{0}-10^{7} \\ B i & d k_{d} / U & \text { Biot number } & 10^{-9}-10^{1} \\ K_{b} & k_{a} C_{C M C} / k_{d} \Gamma_{\infty} & \text { Surfactant solubility parameter } & 10^{-6}-10^{7} \\ \beta_{b} & \Gamma_{\infty} / d C_{C M C} & \text { Surfactant parameter } & 10^{-4}-10^{3} \\ K_{m} & d k_{m} / U & \text { Micelle formation rate } & 10^{-6}-10^{1}\end{array}$

TABle 2. Definition of non-dimensional parameters and range of values they take based on the typical physical parameters in table 1 .

with the following boundary conditions at the channel walls $\boldsymbol{u}_{1}=(0,0), \quad \hat{\boldsymbol{y}} \cdot \nabla C=0, \quad \hat{\boldsymbol{y}} \cdot \nabla M=0 \quad$ at $\quad y=0 \quad$ and $\quad \boldsymbol{u}_{2}=(1,0) \quad$ at $\quad y=1$.

Here, $\hat{\boldsymbol{y}}=(0,1)$ and $\left(r_{1}, r_{2}\right)=(1, r),\left(m_{1}, m_{2}\right)=(1, m)$. At the interface, the fluid velocities have to be equal, i.e.

$$
\boldsymbol{u}_{1}=\boldsymbol{u}_{2} \quad \text { at } \quad y=h(x, t),
$$

and the flux of surfactant is given by

$$
\mathbf{n} \cdot \nabla C=P e_{b} \beta_{b} J_{b} \quad \text { and } \quad \mathbf{n} \cdot \nabla M=0 \quad \text { at } \quad y=h(x, t),
$$

where the unit normal is now defined by $\mathbf{n}=\left(h_{x},-1\right) / \sqrt{1+h_{x}^{2}}$. The evolution of the interface and the interfacial surfactant concentration are described by the following equations (Bassom et al. 2010; Kalogirou 2018)

$$
\begin{gathered}
h_{t}+\left(\int_{0}^{h(x, t)} u_{1} \mathrm{~d} y\right)_{x}=0 \\
\frac{1}{\sqrt{1+h_{x}^{2}}}\left[\left(\sqrt{1+h_{x}^{2}} \Gamma\right)_{t}+\left(\sqrt{1+h_{x}^{2}} u_{I} \Gamma\right)_{x}-\frac{1}{P e_{s}}\left(\frac{\Gamma_{x}}{\sqrt{1+h_{x}^{2}}}\right)_{x}\right]-J_{b}=0
\end{gathered}
$$

where $u_{I}=u_{1}(x, y=h(x, t), t)$. The non-dimensional fluxes $J_{b}$ and $J_{m}$ that appear in equations 2.1c), 2.1d), 2.2c), 2.4 are defined by (see also Edmonstone et al. 2006. 
Craster et al. 2009, Kalogirou \& Blyth 2019)

$$
J_{b}=B i\left(K_{b} C(1-\Gamma)-\Gamma\right), \quad \text { and } \quad J_{m}=K_{m}\left(C^{N}-M\right),
$$

where the first equation describes the flux of monomers to/from the interface from/to the neighbouring bulk fluid due to adsorption/desorption, and the second equation describes the creation of micelles from $N$ monomers.

The remaining interfacial conditions associated with the problem are the dimensionless tangential and normal stress jumps satisfied at the interface $y=h(x, t)$, given by

$$
\begin{array}{r}
{\left[4 m_{i} h_{x} u_{i x}+m_{i}\left(h_{x}^{2}-1\right)\left(u_{i y}+v_{i x}\right)\right]_{2}^{1}=-\frac{\gamma_{x}}{C a} \sqrt{1+h_{x}^{2}}} \\
{\left[-p_{i}\left(1+h_{x}^{2}\right)+2 m_{i}\left(h_{x}^{2} u_{i x}+v_{i y}-h_{x}\left(u_{i y}+v_{i x}\right)\right)\right]_{2}^{1}=\frac{\gamma}{C a} \frac{h_{x x}}{\sqrt{1+h_{x}^{2}}}}
\end{array}
$$

As the system evolves dynamically it induces a non-uniform distribution of surfactant at the interface, which in turn leads to varying interfacial tension and the generation of surface tension gradients at the interface (see, for instance, the right-hand-side term in the tangential stress balance (2.6a)). The dependence of the surface tension on the interfacial surfactant concentration is given by the equation (Chang \& Franses 1995)

$$
\gamma=1+\beta_{s} \ln (1-\Gamma), \quad \text { with } \quad \beta_{s}=M a C a=\frac{\mathcal{R} \mathcal{T} \Gamma_{\infty}}{\gamma_{0}} .
$$

Parameter $\beta_{s}$ is the surfactant elasticity parameter that depends on the ideal gas constant $\mathcal{R}$ and the absolute temperature $\mathcal{T}$.

The problem stated in this subsection is subject to two constraints: the first one is that the overall flow rate through the channel,

$$
Q=\int_{0}^{h} u_{1} \mathrm{~d} y+\int_{h}^{1} u_{2} \mathrm{~d} y
$$

is chosen such that there is a zero pressure drop over a specified domain of length $\mathcal{L}$. The second constraint is that of the conservation of total mass of surfactant, given by

$$
\mathcal{M}=\frac{1}{\mathcal{L}} \int_{0}^{\mathcal{L}} \int_{0}^{h} \frac{1}{\beta_{b}}(C+M) \mathrm{d} y \mathrm{~d} x+\frac{1}{\mathcal{L}} \int_{0}^{\mathcal{L}} \Gamma \mathrm{d} x .
$$

We note that taking the $x$-derivative of both sides in equation $(2.8)$, yields that $Q_{x}=0$ (in view of the velocity continuity conditions $(2.2 b)$ ) which implies that the flow rate can be in general a function of time, i.e. $Q=Q(t)$.

\subsection{Lubrication approximation}

The interest of this study is to follow the spatio-temporal evolution of a given perturbation at the interface, and in particular to explore the effects of surfactants on the emerging nonlinear developments on the interface. In what follows we assume that the wavelength of the disturbance is much larger than the channel height, which suggests a rescaling of the horizontal coordinate and the introduction of a slow time scale as follows

$$
x=\frac{\chi}{\epsilon}, \quad t=\frac{\tau}{\epsilon}, \quad \text { with } \quad \epsilon \ll 1 .
$$

The above change of variables is appropriate since lengths have been scaled earlier with the channel height $d$, which is assumed to be much smaller than the typical wavelength $\lambda$; parameter $\epsilon$ can hence be defined as the height-to-length ratio $\epsilon=d / \lambda \ll 1$. The flow 
velocities and pressures in each fluid $i=1,2$ are expanded in the following manner

$$
u_{i}=\bar{u}_{i}+\tilde{u}_{i}, \quad v_{i}=\epsilon \tilde{v}_{i}, \quad p_{i}=\bar{p}_{i}+\epsilon^{-1} \tilde{p}_{i},
$$

where overbars denote the basic flow - this is assumed to be purely shear driven by the motion of the upper wall and is given by

$$
\begin{array}{ll}
\bar{u}_{1}(y)=s y, & \bar{u}_{2}(y)=\frac{s}{m}(y-1)+1, \\
\bar{p}_{1}(y)=p_{0}-\frac{B o}{C a}\left(y-h_{0}\right), & \bar{p}_{2}(y)=p_{0}-\frac{r B o}{C a}\left(y-h_{0}\right),
\end{array}
$$

with

$$
s=\frac{m}{1+h_{0}(m-1)},
$$

the shear rate, and $p_{0}$ the constant pressure at the undisturbed interface $y=h_{0}$. The scaling for the vertical velocities in 2.11) is such that to provide balance in the continuity equation, while the scaling for the pressure perturbations is necessary to retain capillarypressure contributions at leading order. Assuming that the Reynolds number $R e$ is at most of $O(\epsilon)$ then any inertial effects are negligible in the leading-order perturbation system. The derivation presented in this section follows closely that of Tilley et al. (1994b) for a two-layer channel flow devoid of surfactant, and those of Blyth \& Pozrikidis (2004); Frenkel \& Halpern (2017) for a channel flow with insoluble surfactant at the interface.

The leading-order continuity and momentum equations in each fluid are the lubrication equations, given by

$$
\begin{aligned}
\tilde{u}_{i \chi}+\tilde{v}_{i y} & =0, \\
m_{i} \tilde{u}_{i y y}-\tilde{p}_{i \chi} & =0, \\
\tilde{p}_{i y} & =0,
\end{aligned}
$$

and the leading-order tangential and normal stress balances at the interface $y=h(\chi, \tau)$ are

$$
\begin{aligned}
-\tilde{u}_{1 y}+m \tilde{u}_{2 y} & =\frac{\tilde{M} a \Gamma_{\chi}}{(1-\Gamma)}, \\
-\tilde{p}_{1}+\tilde{p}_{2} & =-(1-r) \frac{\tilde{B} o}{\tilde{C} a}\left(h-h_{0}\right)+\frac{\gamma}{\tilde{C} a} h_{\chi \chi},
\end{aligned}
$$

where the following rescalings for the Bond, capillary and Marangoni numbers have been introduced, $B o=\epsilon^{2} \tilde{B} o, C a=\epsilon^{3} \tilde{C a}, M a=\epsilon^{-1} \tilde{M} a$, in order to retain gravity, surface tension and Marangoni contributions in the leading-order dynamics (recall the surface tension equation of state (2.7)). We note that the parameter scalings considered here are different from those applied in Tilley et al. (1994마), where the capillary number scaling $C a=\epsilon^{2} \tilde{C a}$ is applied, resulting in the surface tension and gravitational effects to appear in the next order.

From $2.13 c$ we have that the pressure perturbations $\tilde{p}_{i}$ are independent of $y$ and hence the momentum equations $2.13 b$ can be integrated in $y$ twice to give

$$
\tilde{u}_{i}(\chi, y, \tau)=\frac{1}{2 m_{i}} y^{2} \tilde{p}_{i \chi}(\chi, \tau)+y a_{i}(\chi, \tau)+b_{i}(\chi, \tau), \quad i=1,2 .
$$

The no-slip boundary conditions at the walls $\tilde{u}_{1}=0$ at $y=0, \tilde{u}_{2}=0$ at $y=1$ are used to determine $b_{1}$ and $b_{2}$, in which case the leading-order perturbations for the horizontal 
velocities become

$$
\begin{aligned}
& \tilde{u}_{1}=\frac{1}{2} y^{2} \tilde{p}_{1 \chi}+y a_{1}, \\
& \tilde{u}_{2}=\frac{1}{2 m}\left(y^{2}-1\right) \tilde{p}_{2 \chi}+(y-1) a_{2} .
\end{aligned}
$$

Similar expressions for the leading-order vertical velocity perturbations can be obtained by the continuity equations 2.13a) integrating in $y$ and using the no normal flow conditions at the walls $\tilde{v}_{1}=0$ at $y=0, \tilde{v}_{2}=0$ at $y=1$ to determine the constants of integration, gives

$$
\begin{aligned}
& \tilde{v}_{1}=-\frac{1}{6} y^{3} \tilde{p}_{1 \chi \chi}-\frac{1}{2} y^{2} a_{1 \chi} \\
& \tilde{v}_{2}=-\frac{1}{6 m}\left(y^{3}-3 y+2\right) \tilde{p}_{2 \chi \chi}-\frac{1}{2}\left(y^{2}-2 y+1\right) a_{2 \chi} .
\end{aligned}
$$

The leading-order normal stress balance $2.14 b$ can be used to write one of the pressure perturbation variables in terms of the other, namely

$$
\tilde{p}_{2}=\tilde{p}_{1}-f, \quad \text { where } \quad f=\frac{1}{\tilde{C} a}\left((1-r) \tilde{B} o\left(h-h_{0}\right)-\gamma h_{\chi \chi}\right),
$$

and the shear stress condition $2.14 a$ can be used to eliminate $a_{2}$ by writing

$$
a_{2}=\frac{1}{m}\left(a_{1}+h f_{\chi}+\frac{\tilde{M} a \Gamma_{\chi}}{(1-\Gamma)}\right) .
$$

What is left is to use the condition of continuity of velocities at the interface $y=h$, which will provide two equations for the remaining unknowns $a_{1}$ and $\tilde{p}_{1 x}$. Continuity of horizontal velocities gives at leading order

$$
\tilde{u}_{2}-\tilde{u}_{1}=\frac{s}{m}(1+(m-1) h)-1, \quad \text { at } \quad y=h,
$$

which can be re-written using (2.16), 2.18), 2.19 to give

$a_{1}=\frac{2(m-s(1+(m-1) h))-\tilde{p}_{1 \chi}\left(1+(m-1) h^{2}\right)+f_{\chi}(h-1)^{2}+2(h-1) \tilde{M} a \frac{\Gamma_{\chi}}{(1-\Gamma)}}{2(1+(m-1) h)}$.

Finally, satisfying continuity of vertical velocities at the interface is equivalent to solving the flow rate equation (2.8), which when using expansions 2.11 becomes

$$
Q=\left(\frac{s}{2 m}\left((m-1) h^{2}+2 h-1\right)+(1-h)\right)+\int_{0}^{h} \tilde{u}_{1} \mathrm{~d} y+\int_{h}^{1} \tilde{u}_{2} \mathrm{~d} y,
$$

where the terms in the bracket come from integrating the basic flow. Equation 2.22 is used to find the leading-order pressure $\tilde{p}_{1 \chi}$ by substituting 2.16 and eliminating $\tilde{p}_{2 \chi}$, $a_{2}$ and $a_{1}$ via (2.18), 2.19) and (2.21), respectively; the final expression for $\tilde{p}_{1 \chi}$ is given by

$$
\begin{aligned}
\tilde{p}_{1 \chi}= & \mathscr{D}^{-1}\left[-6 m\left((m-1) h^{2}-2(m-1) h-1\right)+6 m h(h-1) \frac{\tilde{M} a \Gamma_{\chi}}{(1-\Gamma)}\right. \\
& \left.-(h-1)^{2}\left((m-1) h^{2}+2(1-2 m) h-1\right) f_{\chi}-12 m((m-1) h+1) Q\right],
\end{aligned}
$$


with

$$
\mathscr{D}=(m-1)^{2} h^{4}+4(m-1) h^{3}-6(m-1) h^{2}+4(m-1) h+1 .
$$

Consistent with a previous remark, the flow rate $Q=Q(t)$ is found by satisfying a periodicity condition on the pressure (Ooms et al. 1985, Blyth \& Pozrikidis 2004)

$$
\int_{0}^{\mathcal{L}} \tilde{p}_{1 \chi} \mathrm{d} \chi=\tilde{p}_{1}(\mathcal{L}, t)-\tilde{p}_{1}(0, t)=0,
$$

which fixes the pressure drop in the streamwise direction to be zero. Alternatively, a prescribed volumetric flow rate $Q$ could be considered instead (see Tilley et al. 1994b), but in this case the pressure drop must be determined as part of the solution from $(2.23)$.

Applying the long-wave transformations 2.10 in the bulk and micelle transport equations $(2.1 c)-(2.1 d)$ and boundary conditions $(2.2 a)$ and $(2.2 c)$, results in

$$
\begin{gathered}
C_{\tau}+\left(\bar{u}_{1}+\tilde{u}_{1}\right) C_{\chi}+\tilde{v}_{1} C_{y}=\frac{1}{\tilde{P} e_{b}}\left(C_{\chi \chi}+\frac{1}{\epsilon^{2}} C_{y y}\right)-\tilde{J}_{m}, \\
M_{\tau}+\left(\bar{u}_{1}+\tilde{u}_{1}\right) M_{\chi}+\tilde{v}_{1} M_{y}=\frac{1}{\tilde{P} e_{m}}\left(M_{\chi \chi}+\frac{1}{\epsilon^{2}} M_{y y}\right)+\tilde{J}_{m}, \\
C_{y}=0 \text { and } M_{y}=0 \text { at } y=0, \\
-\frac{1}{\epsilon^{2}} C_{y}+h_{\chi} C_{\chi}=\tilde{P}_{b} \beta_{b} \tilde{J}_{b} \text { and }-\frac{1}{\epsilon^{2}} M_{y}+h_{\chi} M_{\chi}=0 \text { at } y=h(\chi, \tau),
\end{gathered}
$$

with the scaled fluxes given by

$$
\tilde{J}_{b}=\tilde{B} i\left(K_{b} C(1-\Gamma)-\Gamma\right) \quad \text { and } \quad \tilde{J}_{m}=\tilde{K}_{m}\left(C^{N}-M\right) .
$$

Here, some further parameter rescalings have been introduced by $B i=\epsilon \tilde{B i}, K_{m}=\epsilon \tilde{K}_{m}$, $P e_{b}=\epsilon \tilde{P} e_{b}, P e_{m}=\epsilon \tilde{P} e_{m}$. At leading order in $\epsilon$, system 2.25 is simplified to $C_{y y}=0$, $M_{y y}=0$, with $C_{y}=0, M_{y}=0$ at $y=0$ and $y=h$, which gives that the solutions at leading order are independent of the vertical coordinate $y$. The following expansions can therefore be introduced

$$
\begin{aligned}
C(\chi, y, \tau) & =C_{0}(\chi, \tau)+\epsilon^{2} \tilde{P} e_{b} C_{1}(\chi, y, \tau)+\cdots \\
M(\chi, y, \tau) & =M_{0}(\chi, \tau)+\epsilon^{2} \tilde{P} e_{m} M_{1}(\chi, y, \tau)+\cdots
\end{aligned}
$$

We define the following notation for the average of a quantity $\mathcal{F}$ in the lower fluid,

$$
[[\mathcal{F}]]=\frac{1}{h} \int_{0}^{h} \mathcal{F}(\chi, y, \tau) \mathrm{d} y,
$$

and note that the average of the perturbations $C_{1}$ and $M_{1}$ is taken to be zero (Jensen \& Grotberg 1993), i.e.

$$
\left[\left[C_{1}\right]\right]=0, \quad\left[\left[M_{1}\right]\right]=0 .
$$

Expansions (2.27) are equivalent to assuming a rapid vertical diffusion of surfactant in the bulk (Jensen \& Grotberg 1993, Craster et al. 2009). These are subsequently substituted into system (2.25) and the resulting leading-order transport equations for the bulk and micelle concentrations are integrated across the lower fluid; upon use of the leading-order boundary conditions at the wall and interface to eliminate the perturbation variables $C_{1}$, 
$M_{1}$, the final evolution equations emerge and are given by

$$
\begin{aligned}
& C_{0 \tau}+\left[\left[u_{1}\right]\right] C_{0 \chi}-\frac{\left(h C_{0 \chi}\right)_{\chi}}{h \tilde{P} e_{b}}+\frac{\beta_{b} \tilde{J}_{b 0}}{h}+\tilde{J}_{m 0}=0, \\
& M_{0 \tau}+\left[\left[u_{1}\right]\right] M_{0 \chi}-\frac{\left(h M_{0 \chi}\right)_{\chi}}{h \tilde{P} e_{m}}-\tilde{J}_{m 0}=0,
\end{aligned}
$$

and

$$
\tilde{J}_{b 0}=\tilde{B} i\left(K_{b} C_{0}(1-\Gamma)-\Gamma\right) \quad \text { and } \quad \tilde{J}_{m 0}=\tilde{K}_{m}\left(C_{0}^{N}-M_{0}\right)
$$

A similar equation to $2.30 a$ for the bulk concentration has been derived by Jensen \& Grotberg (1993) in the absence of micelles, but the horizontal velocity was approximated by a leading-order solution of the momentum equations (also, their system was for one fluid only). Furthermore, Mavromoustaki et al. (2012a b $)$ derived the same equations in their studies of the dynamics of a climbing surfactant-laden film. We note that the leading-order monomer and micelle concentrations in the bulk are advected by the average of the horizontal velocity in the lower fluid, and the vertical structure of the velocity field does not affect the bulk concentrations at leading order. Consequently the above averaged equations cannot capture convective effects within the bulk fluid.

The first-order bulk and micelle concentration distributions across the bulk fluid can be determined by subtracting $2.30 a), 2.30 b$ from the leading-order system obtained after expansions (2.27) are substituted into 22.25) (Jensen \& Grotberg 1993), and integrating in $y$ twice while using the boundary conditions in $(2.25 c)-(2.25 d)$ at leading order. The resulting first-order concentrations are

$$
\begin{aligned}
C_{1} & =\left(\iint u_{1}(\hat{y}) \mathrm{d} \hat{y} \mathrm{~d} y-\frac{1}{2} y^{2}\left[\left[u_{1}\right]\right]\right) C_{0 \chi}+\left(\frac{h_{\chi} C_{0 \chi}}{\tilde{P} e_{b}}-\beta_{b} \tilde{J}_{b 0}\right) \frac{y^{2}}{2 h}+d_{c}, \\
M_{1} & =\left(\iint u_{1}(\hat{y}) \mathrm{d} \hat{y} \mathrm{~d} y-\frac{1}{2} y^{2}\left[\left[u_{1}\right]\right]\right) M_{0 \chi}+\frac{h_{\chi} M_{0 \chi}}{\tilde{P} e_{m}} \frac{y^{2}}{2 h}+d_{m} .
\end{aligned}
$$

The constants of integration $d_{c}$ and $d_{m}$ can be calculated using the zero-average conditions 2.29 for $C_{1}$ and $M_{1}$.

Finally, the leading-order kinematic equation 2.3 and leading-order transport equation for interfacial surfactant 2.4 are given by

$$
h_{\tau}+\left(\frac{1}{2} s h^{2}+\frac{1}{2} a_{1} h^{2}+\frac{1}{6} \tilde{p}_{1 \chi} h^{3}\right)_{\chi}=0,
$$

and

$$
\Gamma_{\tau}+\left(\left.u_{1}\right|_{y=h} \Gamma\right)_{\chi}-\frac{1}{\tilde{P}_{e}} \Gamma_{\chi \chi}-\tilde{J}_{b 0}=0
$$

where the scaled Péclet number is introduced by $P e_{s}=\epsilon \tilde{P} e_{s}$. These two equations together with the two transport equations in 2.30 for the bulk and micelle concentrations form a system of evolution equations that describe the leading-order dynamics of the problem considered. This system needs to be solved to study the spatio-temporal evolution of the fluid interface and the surfactant concentrations, making sure that the leading-order total mass of surfactant

$$
\mathcal{M}_{0}=\frac{1}{\mathcal{L}} \int_{0}^{\mathcal{L}}\left(\frac{1}{\beta_{b}}\left(C_{0}+M_{0}\right) h+\Gamma\right) \mathrm{d} \chi,
$$

remains constant. Once the leading-order system of equations $\sqrt{2.32}, \sqrt{2.33}, \sqrt{2.30}$ is solved and solutions for $h, \Gamma, C_{0}, M_{0}$ are obtained, then fluid velocities $u_{i}, v_{i}, i=$ 
1,2 , can be found from (2.16), 2.17) and the concentration perturbations $C_{1}, M_{1}$ can be calculated from (2.31). In summary, the lubrication model presented in this section considers the following parameter rescalings

$$
\begin{aligned}
& B o=\epsilon^{2} \tilde{B} o, \quad C a=\epsilon^{3} \tilde{C} a, \quad M a=\epsilon^{-1} \tilde{M} a, \quad B i=\epsilon \tilde{B} i, \quad K_{m}=\epsilon \tilde{K}_{m}, \\
& P e_{s}=\epsilon \tilde{P} e_{s}, \quad P e_{b}=\epsilon \tilde{P} e_{b}, \quad P e_{m}=\epsilon \tilde{P} e_{m} \text {. }
\end{aligned}
$$

Finally, we note that by reverting back to the original parameters and variables, we obtain equations 2.32, 2.33, 2.30) with $x, t$ instead of $\chi, \tau$ and the parameters in 2.35 without the tildes.

The asymptotic model derived in this section based on the parameter scalings in 2.35 should be appropriate for a range of physical situations and/or experiments. A multi-layer system such as a water-oil system (with the aqueous phase being populated with surfactants) will have the fluid characteristics (densities, viscosities, surface tension) displayed in table 1 (Shen et al. 2002; Barthelet et al. 1995; Pereira \& Kalliadasis 2008). Different types of surfactants can be used, for example sodium dodecyl sulfate (SDS) or isopropanol (Georgantaki et al. 2016). According to Chang \& Franses (1995), the maximum packing concentration $\Gamma_{\infty}$ does not vary significantly among different types of surfactants, while the sorption kinetic ratio $k_{a} / k_{d}$ (and thus non-dimensional parameter $K_{b}$ ) can show large variations - for instance, a large value of $K_{b}$ signifies a surfactant that is physically more surface active.

Based on the physical properties given in table 1, the non-dimensional parameters take values in the ranges shown in table 2. In an experimental set-up where a thin and long channel is used (Barthelet et al. 1995), here assumed to have a height as small as $1 \mu \mathrm{m}$ in order to capture the microfluidics regime, the Reynolds number is typically negligible for small shear or flow rates. In such scenarios where a large length-to-height aspect ratio is valid, the scalings adopted in 2.35) are applicable, i.e. the values of the Bond and capillary numbers are typically minuscule, the Marangoni number can be relatively large, and parameters $B i$ and $K_{m}$ are usually small. Owing to the small-scale diffusion rates, the Péclet numbers are typically very large but there are some practical scenarios when smaller Péclet values are relevant (e.g. for channel gap heights in the micrometer range). Even though the model was derived based on the assumption of small Péclet numbers, in the computations later the three Péclet numbers take moderate values that push the model slightly beyond its formal range of validity, in order to get some physically relevant cases. Finally, the effects of gravity can be ignored by selecting fluids of similar densities, i.e. with a density ratio $r \approx 1$.

\section{Numerical simulations}

\subsection{Numerical methods}

The four governing equations 2.32, 2.33), 2.30 are solved in a bounded interval $x \in[-L, L]$ with periodic boundary conditions (in essence we take the length $\mathcal{L}=2 L$ ). The spatial derivatives are therefore evaluated using Fast Fourier Transforms (FFT) in Matlab (Trefethen 2000). The system is written in the form $\boldsymbol{U}_{t}+\boldsymbol{F}(\boldsymbol{U})=\mathbf{0}$, with vector $\boldsymbol{U}=\left(h, \Gamma, C_{0}, M_{0}\right)$ and nonlinear operator $\boldsymbol{F}(\boldsymbol{U})$. The interval $[-L, L]$ is partitioned into a uniform mesh with $2 N_{m}$ points, defined by $x_{n}=-L+n \Delta x$ with $\Delta x=L / N_{m}$ and $n=0,1, \ldots, 2 N_{m}-1$, and the solution for $\boldsymbol{U}$ at each mesh point is approximated by a value $\boldsymbol{U}_{n}$. The time discretisation scheme is based on the theta-method, which uses a weighted average of the solution in the nonlinear term $\boldsymbol{F}(\boldsymbol{U})$ and the system solved is 
given by

$$
\frac{\boldsymbol{U}_{n+1}-\boldsymbol{U}_{n}}{\mathrm{~d} t}-\boldsymbol{F}\left(\theta \boldsymbol{U}_{n+1}+(1-\theta) \boldsymbol{U}_{n}\right)=0,
$$

where $0 \leqslant \theta \leqslant 1$. Taking $\theta=1$ gives the implicit Euler method which is first-order accurate in time, while for $\theta=0$ the scheme is explicit and requires much smaller time steps compared to other values of $\theta$. Here we will use the theta-method with $\theta=1 / 2$, which is unconditionally stable and is second-order accurate in time.

The nonlinear system is solved starting from initial conditions

$$
h(x, 0)=h_{0}+h_{a} \cos \left(\frac{K \pi x}{L}\right), \quad \Gamma(x, 0)=\bar{\Gamma}, \quad C_{0}(x, 0)=\bar{C}, \quad M_{0}(x, 0)=\bar{M},
$$

where $\bar{\Gamma}, \bar{C}, \bar{M}$ denote the equilibrium surfactant concentrations; the initial conditions (3.2) hence correspond to a perturbed interface (with perturbation of amplitude $h_{a}$ and $K$ waves in one period) with uniform surfactant concentrations. Typically the initial concentration at the interface $\bar{\Gamma}$ is prescribed and the equilibrium concentrations for the monomers and micelles in the bulk, $\bar{C}$ and $\bar{M}$, are calculated by setting the fluxes in (2.5) to zero, yielding

$$
\bar{C}=\frac{\bar{\Gamma}}{K_{b}(1-\bar{\Gamma})}, \quad \bar{M}=\bar{C}^{N} .
$$

The overall surfactant mass in equilibrium can hence be calculated by

$$
\mathcal{M}_{0}=\frac{h_{0}}{\beta_{b}}(\bar{C}+\bar{M})+\bar{\Gamma} .
$$

We note that in what follows, whenever we refer to being below the CMC this means that the selected values for $\bar{C}+\bar{M}<1$, i.e. less than CMC in dimensional units.

\subsection{Linear growth rates}

To validate the numerical code we perform comparisons between growth rates found using linear stability analysis and numerical computations. Introducing a normal-mode perturbation to the steady state $\left(h, \Gamma, C_{0}, M_{0}\right)=\left(h_{0}, \bar{\Gamma}, \bar{C}, \bar{M}\right)$ of the form $h=h_{0}+$ $\delta \hat{h} \mathrm{e}^{i k x+\sigma t}$, etc. for small perturbation amplitude $\delta \ll 1$, leads to a linearised system coming from 2.32, 2.33, 2.30. Here, $\sigma$ is generally complex, $k$ is real, and quantities with hat decorations are the perturbation eigenfunctions. The resulting dispersion relation is a fourth-order polynomial for $\sigma$ which is solved to find four growth rates given by the real part $s=\Re(\sigma)$ (the polynomial in $\sigma$ is of third order when the concentrations are below the $\mathrm{CMC}$ and the micelle equation is ignored). Out of the four growth rates, two are seen to pass through $k=0$ and the other two (corresponding to the bulk and micelle surfactant modes) are negative at $k=0$. Typically one of the four modes is unstable, with the instability manifesting itself for a range of wavenumbers $0<k<k_{c}$, where $k_{c}$ is the cut-off wavenumber. The growth rates obtained are found to be in excellent agreement with the results of Kalogirou \& Blyth (2019) who solved the Orr-Sommerfeld eigenvalue problem for arbitrary wavelength perturbations; the good agreement is verified for all cases shown in this study as long as $k$ is not too large - this is expected since the model is derived assuming that the channel length-to-height ratio is large, i.e. any interfacial waves will be large-wavelength waves. The third mode (and fourth mode if the bulk concentration is above the CMC) only agrees with the numerical solution of the full system (as explained in Kalogirou \& Blyth (2019) using the Chebyshev collocation method (Orzag 1971)) when $P e_{b}, P e_{m}$ are relatively small. Typical curves for the dominant growth rate are shown in figure 2 panel (a) demonstrates three growth rates for decreasing values of surfactant 


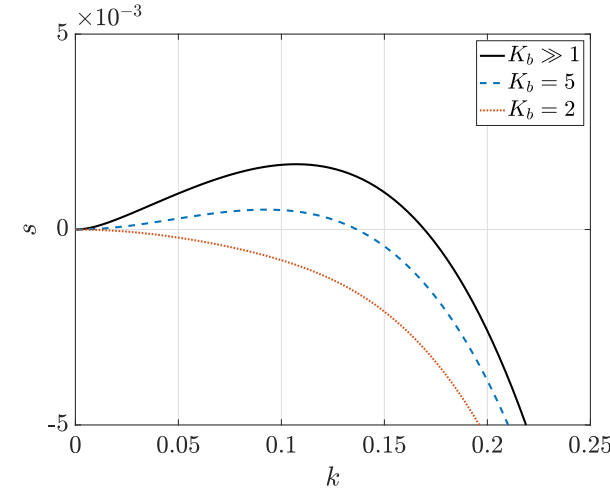

(a) Below the CMC

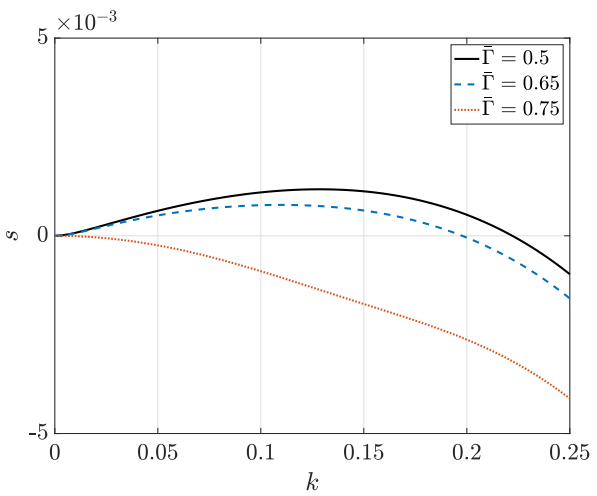

(b) Above the CMC

FiguRE 2. Dominant growth rate curves found from linear stability analysis of the lubrication model for different values of (a) solubility parameter $K_{b}$, or (b) equilibrium interfacial concentration $\bar{\Gamma}$. In panel (a) the parameter set $h_{0}=0.4, m=0.5, \bar{\Gamma}=0.5$ is used, while panel (b) uses the values $h_{0}=0.2, m=0.5, K_{b}=3$. The rest of the parameters take the values $B o=0, C a=0.001, M a=10, B i=0.1, \beta_{b}=1, K_{m}=0.1, N=10, P e_{s}=P e_{b}=P e_{m}=10$.

solubility $K_{b}$, where it is seen that a sufficient amount of solubility stabilises the system. Panel (b) shows a case with increasing initial concentration $\bar{\Gamma}$ (or equivalently increasing the total available surfactant mass) and the system is seen to be stabilised when the bulk concentration grows beyond the CMC (this can be calculated from $\sqrt{3.3}$ for the given values of $\left.\bar{\Gamma}, K_{b}, N\right)$.

To enable comparison with the linear stability results, we solve the fully nonlinear system using initial conditions $(3.2)$ with perturbation amplitude $h_{a} \ll 1$. At small times and before the solution saturates to a nonlinear state (but after any initial transients), the solution remains in the linear regime and is of the form $h=h_{0}+h_{a} \mathrm{e}^{i k x+\sigma t}$, where $\sigma=\sigma_{r}+i \sigma_{i}$ is the complex growth rate and $k$ is the wavenumber. Taking the logarithm of the $\mathcal{L}_{2}$-norm gives $\log \left(\left\|h-h_{0}\right\|_{2} / h_{a}\right)=\sigma_{r} t+\mathcal{C}$ for some constant $\mathcal{C}$. Therefore by plotting this logarithmic expression we obtain a line of slope $\sigma_{r}$; this allows us to compare the amplification rate $\sigma_{r}$ with the prediction of the dispersion curve $s$ found from linear stability analysis.

We perform a number of comparisons between growth rates found from linear theory and the corresponding growth rates obtained from nonlinear simulations as described above. The (real) wavenumber $k$ and integer frequency $K$ are connected through the relationship $k=\pi K / L$ - this means that a perturbation of wavelength $2 \pi / k$ on the real line corresponds to a perturbation of wavelength $2 L / K$ in the periodic domain. For a given set of parameters, we either fix the domain half-length $L$ and select a perturbation frequency $K$ such that $k=\pi K / L<k_{c}$ (the critical $k_{c}$ is found from the linear dispersion curve), or we fix the frequency $K$ and choose a domain length satisfying $L>L_{c}=\pi K / k_{c}$. The growth rate predictions from linear stability theory and nonlinear computations in the linear regime are found to have good agreement for all tested cases.

\subsection{Nonlinear solutions}

This section presents a range of results obtained from time-dependent calculations of the governing system of equations 2.32 , 2.33 , 2.30 , aiming to identify the effect of surfactant solubility on the dynamics of the system. To determine the impact of solubility on the solutions, we use the following norm as a measure of the interfacial wave amplitude, 
defined by

$$
\left\|h-h_{0}\right\|^{2}=\frac{1}{2 L} \int_{-L}^{L}\left(h-h_{0}\right)^{2} \mathrm{~d} x,
$$

and computed using the trapezoidal quadrature rule. Mass conservation was verified in all numerical computations, by calculating the total surfactant mass using (2.34) and ensuring that it remains constant (to within a small absolute error typically of the order of $\left.10^{-8}\right)$. The wave speed of interfacial travelling waves found as solutions can be obtained by writing $h(x, t)=h(z)$ with $z=x-c t$ (similarly for the rest of the variables), and using equation $(2.33)$ to obtain the following formula

$$
c=\frac{\int_{-L}^{L}\left(\left(\left.u_{1}\right|_{y=h} \Gamma\right)_{z} \Gamma_{z}-J_{b 0} \Gamma_{z}\right) \mathrm{d} z}{\int_{-L}^{L} \Gamma_{z}^{2} \mathrm{~d} z} .
$$

All the results presented in this section are obtained by considering a frame of reference moving with the travelling wave.

It should be noted that, once the interfacial height $h(x, t)$ is determined, then the leading-order flow perturbations throughout the channel follow from equations (2.16), (2.17), 2.18), 2.23). Such expressions can be used to calculate the vorticity distribution in the channel and also to construct streamlines. The streamlines are formulated by writing the fluid velocity in each fluid layer in terms of the streamfunction $\Psi_{i}(x, y, t)$ such that $u_{i}-c=\Psi_{i y}, v_{i}=-\Psi_{i x}$, for $i=1,2$. Here, by subtracting the interfacial travelling wave speed $c$ from the horizontal velocity we consider a frame of reference in which the interface is stationary and corresponds to a streamline. The streamfunction in the lower fluid is found to be (the one in the upper layer is not given but it can be found in a similar way)

$$
\Psi_{1}(x, y, t)=\frac{1}{2} s y^{2}+\frac{1}{2} a_{1} y^{2}+\frac{1}{6} \tilde{p}_{1 \chi} y^{3}-c y,
$$

with the constant of integration determined such that $\Psi_{1}=0$ on the lower wall $y=0$.

In what follows we fix the domain half-length to $L=28$ and the perturbation frequency to $K=1$ (note that for this choice of domain length, only perturbations of this particular frequency are unstable - larger domain lengths can support a spectrum of unstable frequencies and more complex dynamics, as shown in a related study for insoluble surfactant by Kalogirou \& Papageorgiou (2016)). Computations are performed with initial perturbation amplitude equal to $20 \%$ of the total undisturbed thickness of the lower fluid, i.e. $h_{a}=h_{0} / 5$, and the final time is taken to be large enough so that the solutions saturate to a coherent structure (typically around $t=5000$ ). Even though the asymptotic parameter $\epsilon$ has been scaled out of the final lubrication model, the model is only valid if the scalings in 2.35 are satisfied. Therefore the parameter $\epsilon$ is kept to a fixed value $\epsilon=0.1$ and the rest of the parameters that scale with $\epsilon$ take the values $B o=0, C a=\epsilon^{3}=0.001, M a=\epsilon^{-1}=10, B i=\epsilon=0.1, K_{m}=\epsilon=0.1, P e_{s}=100 \epsilon=10$, $P e_{b}=100 \epsilon=10, P e_{m}=100 \epsilon=10$. Moreover, the undisturbed interfacial surfactant concentration is fixed to $\bar{\Gamma}=0.5$ (unless otherwise stated), the micelle size is taken to be $N=10$ and the solubility parameter is fixed to $\beta_{b}=1$. Based on the selected value of $\bar{\Gamma}$, the equilibrium concentrations $\bar{C}$ and $\bar{M}$ are calculated from 3.3 . We note that the same saturated wave can be reached with different initial distributions of the same total available surfactant mass (for example, initially all the surfactant could be at the interface or in the bulk).

We focus the numerical investigation on four distinct cases: in the first two the bulk concentrations are below the CMC and the parameter sets are chosen to support 
(in)stability when the surfactant is insoluble - the strength of surfactant solubility is then increased to determine its influence on the stability of the interface. In particular, the parameters in the first (second) case satisfy the condition $m<n^{2}\left(m \geqslant n^{2}\right)$, for which the system with insoluble surfactant has been shown to be unstable (stable) (Frenkel \& Halpern 2002; Halpern \& Frenkel 2003). The third case considers the effect of total mass of surfactant on the stability, especially in cases where bulk concentration is above the CMC and micelles are also formed. In all the above cases, the fluid densities are taken to be equal (i.e. $r=1$ ) in order to eliminate density stratification effects and focus on the effect of surfactants and their solubility. A fourth and final case considers a flow with surfactants at high concentrations above the CMC that is susceptible to the Rayleigh-Taylor instability due to unstable density stratification (for related studies on the influence of gravity in a two-layer channel flow with insoluble surfactants we refer the reader to Frenkel \& Halpern (2017); Kalogirou (2018)). The aim is to identify the dominant physical effect in a scenario where surfactants and gravity are interacting.

\subsubsection{Below the CMC for $m<n^{2}$}

We select a parameter set that leads to an unstable interface in the case when the surfactant is mostly attracted to the interface (i.e. insoluble or nearly insoluble), and then reduce the value of the solubility parameter $K_{b}$ to identify its effect on the stability. The undisturbed interfacial height is at $h_{0}=0.4$, the viscosity ratio is $m=0.5$ and the equilibrium concentration of interfacial surfactant is $\bar{\Gamma}=0.5$. Here the chosen parameters satisfy the condition $m<n^{2}$ (where $n$ is the fluids thickness ratio equal to $\left.n=\left(1-h_{0}\right) / h_{0}=1.5\right)$, and therefore the insoluble system is unstable (Frenkel \& Halpern 2002). Further information about the stability of the system for the selected parametric set can be gathered from the growth rate curves in figure $2 \mathrm{a}$ when looking at the specific wavenumber $k=\pi / 28=0.1122$ corresponding to a domain of length $L=28$ as considered here; in particular, it is anticipated that for sufficiently strong surfactant solubility effects the interface will be stabilised.

The above predictions from linear stability analysis are followed into the nonlinear regime through simulations of the present nonlinear lubrication model. An initial disturbance of the form 3.2 is allowed to evolve in time and it eventually saturates to a nonlinear travelling wave at large times (typically after about 3000 time units). Panel (a) in figure 3 demonstrates the saturated interfacial wave for the case when the surfactant is predominantly attracted to the interface (equivalently $K_{b} \gg 1$ ). The thick black line shows the location of the interface while the thin black lines within the two fluids are the streamlines, plotted as the contour lines of the respective streamfunction calculated from (3.7). The streamlines indicate the presence of an eddy in the lower fluid that is centred around the point $(x, y)=(-4.6,0.364)$ and spans the whole domain - in fact, a stagnation point exists directly below the interfacial wave trough and within the lower fluid around $(x, y)=(19.5,0.315)$. At the initial stages of the evolution the streamlines under the wave peak intersect the interface, but after some initial transient (up to $t \approx 300$ ) the eddy appears to take its final form (as seen in figure 3 a) and the streamlines immediately above the eddy are parallel with the interface. In the frame of reference moving with the interfacial travelling wave, the interface and the fluid directly below it are both moving from left to right, the eddy is rotating clockwise (evident by the colour in figure 3 a corresponding to the vertical velocity in the lower fluid $v_{1}$ ) and the fluid beneath the eddy is moving from right to left. We note that the existence of eddies under a deforming interface has also been reported in related thin film flows (Blyth et al. 2018), but those eddies were trapped inside the main part of solitary waves and appeared only when the wave amplitude was sufficiently large. Here we find that the eddy gets longer for waves 


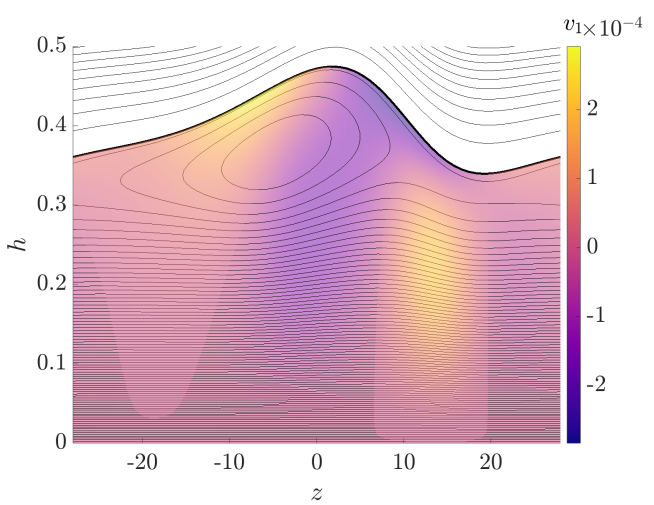

(a) $K_{b} \gg 1$

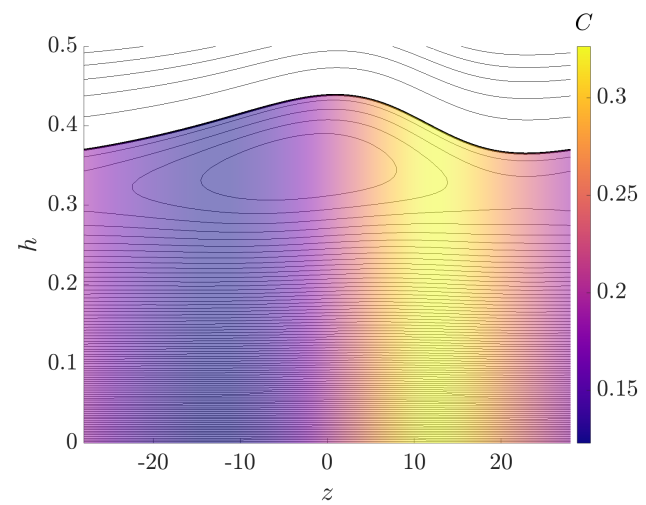

(b) $K_{b}=5$

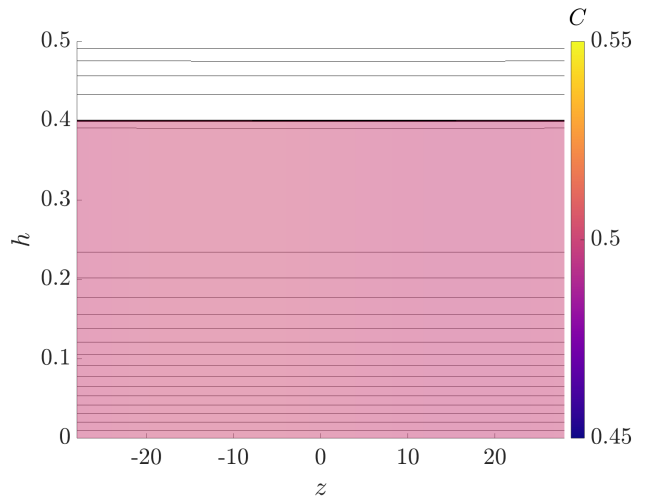

(c) $K_{b}=2$

FiguRE 3. Interfacial deformation (thick black line) for three values of solubility parameter $K_{b}$, demonstrating stabilisation of the interface for sufficiently strong surfactant solubility. The thin black lines shown within the fluids are the streamlines which are equally spaced in $\Psi_{1}, \Psi_{2}$, and the colours in each figure correspond to the (a) vertical velocity $v_{1}$, or (b,c) bulk surfactant concentration $C$. The parameter values used are $h_{0}=0.4(n=1.5)$ and $m=0.5$, which satisfy the condition $m<n^{2}$ according to which the system with insoluble surfactant (panel (a)) is unstable. The rest of the parameters are the same as in figure $2 \mathrm{a}$ and $\epsilon=0.1$. The time evolution of the interfacial wave and interfacial surfactant concentration can be seen in Videos 1-3 provided as supplementary material.

of smaller saturated amplitude; in fact, for wave amplitudes smaller than $10^{-2}$ the eddy gets elongated as time evolves, the enclosed streamlines eventually detach and the eddy disappears (at sufficiently large times but before the wave reaches saturation).

When the surfactant is weakly soluble with $K_{b}=5$ (figure $3 \mathrm{p}$ ), the system is still unstable but the saturated interfacial wave has a smaller amplitude and is seen to be smoother compared to the insoluble case (panel (a)). The colour shown in panel (b) demonstrates the bulk surfactant concentration $C$ (calculated using the first two terms in $(2.27 a)$ ), which is seen to be uniform in the vertical and to attain its maximum concentration ahead of the location where the lower fluid is the thickest. When the surfactant solubility effects are sufficiently strong, i.e. for a small enough value of $K_{b}=2$, a complete stabilisation of the flow is observed characterised by uniform fluid thicknesses and constant surfactant concentrations throughout the channel as shown in figure 3 . The observed stabilisation due to surfactant solubility is summarised in figure 4 a where the solution norms 3.5 are depicted for the three cases discussed above. 


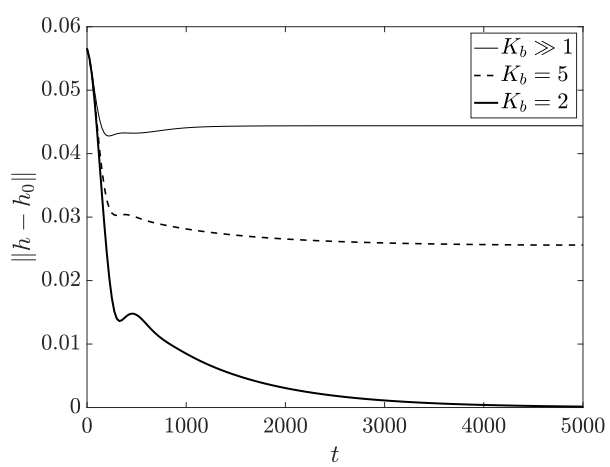

(a) $m=0.5$

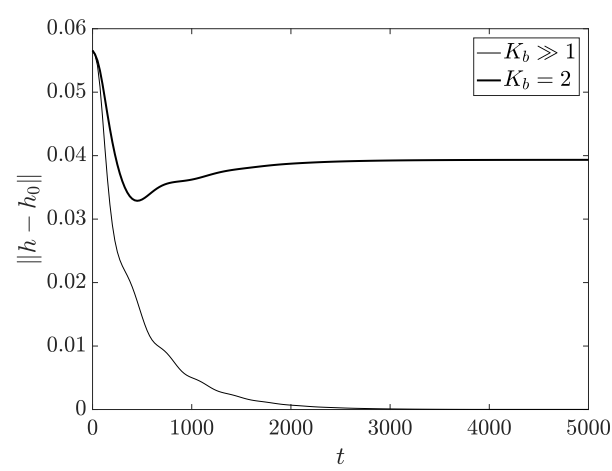

(b) $m=5.0$

FiguRE 4. Norms of interfacial displacement for different values of solubility parameter $K_{b}$, corresponding to the simulations presented in (a) figure 3 and (b) figure 5.

\subsubsection{Below the CMC for $m \geqslant n^{2}$}

Similarly to the previous case, we consider an undisturbed interfacial thickness $h_{0}=0.4$ $(n=1.5)$ but this time the viscosity ratio is larger at $m=5$, satisfying $m \geqslant n^{2}$. The system with insoluble surfactant is hence expected to be stable (Frenkel \& Halpern 2002), as confirmed by panel (a) of figure 5 where a flat interface is seen and the flow is unidirectional (see also the corresponding solution norm in figure $4 \mathrm{~b}$ which is seen to tend to zero at large times). When the surfactant is sufficiently soluble the system can be destabilised, as demonstrated by the saturated travelling wave in panel (b) of figure 5 which is obtained for $K_{b}=2$. Note that for the choice of parameters used here, the equilibrium concentration of monomers in the bulk is $\bar{C}=0.5$ which is way below the critical micelle concentration (equal to 1 in dimensionless units). However, what seems to be particularly interesting in the result of figure $5 \mathrm{~b}$, is that a small concentration of micelles is developed within the lower fluid. The micelle concentration (calculated using the first two terms in $(2.27 b$ ) ) is illustrated with colour in panel (b); it can be observed that the micelles are mainly formed under the wave crest whereas their concentration remains zero in the rest of the domain. As the system develops dynamically, the nonlinear fluxes $J_{b}$ and $J_{m}$ (see (2.5) ) depart from the initial equilibrium state and a non-uniform distribution of surfactant occurs due to interface deformation and the ensuing Marangoni flow. This leads the saturated monomer concentration to reach a value of 0.9 in the vicinity of the peak of the interfacial wave (not shown here) and the micelle concentration to reach a value of about 0.27 , and therefore the CMC is exceeded. In this scenario it is expected that the nonlinear equation of state for the surface tension (2.7) becomes more important. A similar result has been observed in Craster et al. (2009) in their study of the breakup of surfactant-laden jet.

\subsubsection{Above the $C M C$}

Increasing the total available mass of surfactant increases the concentration in the bulk until eventually the CMC is exceeded and micelles start to form. When this happens it is expected that the flow will be stable due to the weakening of the Marangoni forces which is a consequence of the micellisation (Kalogirou \& Blyth 2019). This remark is also corroborated by the growth rate curves in figure $2 \mathrm{~b}$, when looking at the wavenumber $k=\pi / 28=0.1122$ which corresponds to $L=28$. Figure 6 demonstrates the results of numerical calculations with $h_{0}=0.2, m=0.5$ and two different values of overall surfactant mass $\mathcal{M}_{0}$, corresponding to bulk concentrations below and above the CMC. 


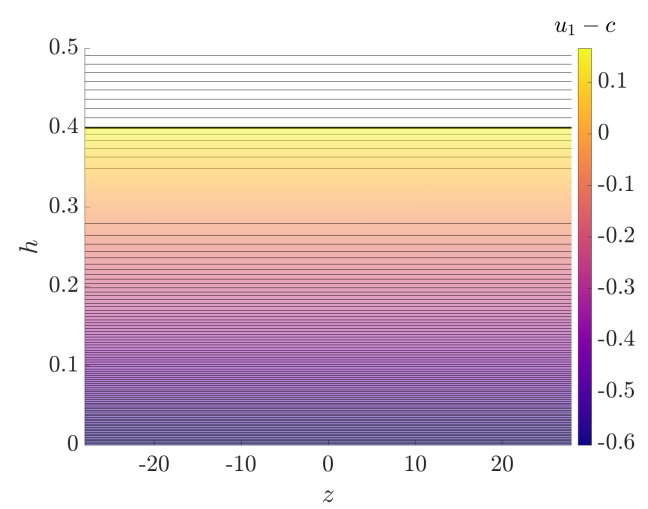

(a) $K_{b} \gg 1$

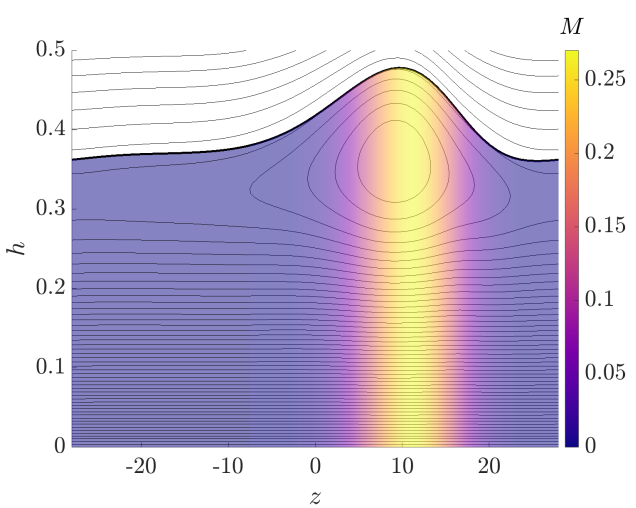

(b) $K_{b}=2$

FiguRE 5. Interfacial deformation (thick black line) for two values of solubility parameter $K_{b}$, demonstrating destabilisation of the interface for sufficiently strong surfactant solubility. The thin black lines shown within the fluids are the streamlines which are equally spaced in $\Psi_{1}, \Psi_{2}$, and the colours in each figure correspond to (a) the horizontal velocity $u_{1}-c$, or (b) the micelle concentration $M$. The parameter values used are $h_{0}=0.4(n=1.5)$ and $m=5$, which satisfy the condition $m \geqslant n^{2}$ according to which the system with insoluble surfactant (panel (a)) is stable. The rest of the parameters are the same as in figure $2 \mathrm{a}$ and $\epsilon=0.1$. The time evolution of the interfacial wave and interfacial surfactant concentration can be seen in Videos 4-5 provided as supplementary material.

In particular, panels $(\mathrm{a}, \mathrm{b})$ in figure 6 consider an initial interfacial concentration $\bar{\Gamma}=0.5$, in which case the initial bulk and micelle concentrations are $\bar{C}=1 / 3, \bar{M}=1 / 3^{10} \approx 1.7 \times$ $10^{-5}$ (calculated from $\left(3.3\right.$ ) ) and the total surfactant mass is $\mathcal{M}_{0}=0.5667$ (calculated from (3.4). Panels (c,d) use $\bar{\Gamma}=0.75$, hence we have $\bar{C}=1, \bar{M}=1$ and a total surfactant mass of $\mathcal{M}_{0}=1.15$.

For the chosen parametric set, the corresponding insoluble system is unstable irrespective of the value of $\bar{\Gamma}$ (since $m<16=n^{2}$ ) and a solitary-type pulse is seen to emerge at large times (panels (a) and (c)). An eddy is developed in the lower fluid which is rotating clockwise similarly to the results reported above in section 3.3.1 (the colour in panels (a,c) denotes the vertical velocity in the fluid). When the surfactant is soluble with $K_{b}=3$, then the interface continues to be unstable if the surfactant concentration is relatively low but the solitary pulse has a smaller amplitude, see panel (b) which employs $\bar{\Gamma}=0.5$. A small concentration of micelles is also created at the front edge of the eddy, as shown by the colour in figure $6 \mathrm{~b}$, similarly to the result in figure $5 \mathrm{p}$. On the other hand, for higher bulk concentrations beyond the CMC the interface is stable and the micelle concentration is uniform at $M=1$, as illustrated in panel (d) for $\bar{\Gamma}=0.75$. The corresponding solution norms for the calculations discussed in this section can be found in figure 7, confirming the reduction in the saturated wave amplitude due to surfactant solubility effects for concentrations below the CMC (top), and the flow stabilisation due to micellisation when the bulk concentration is above the CMC (bottom).

\subsubsection{Unstably stratified system}

In the previous section it was shown that once the surfactant concentration in the bulk exceeds the CMC, then the flow is stable. This behaviour is anticipated for an inertialess stably stratified flow with constant surface tension. The aim of this case study is to examine the flow dynamics due to the interacting effects of gravity and surfactants at high concentrations above the CMC. We therefore consider the flow analysed above in section 3.3.3. which has been found to be stable for equal-density fluids, and we introduce 


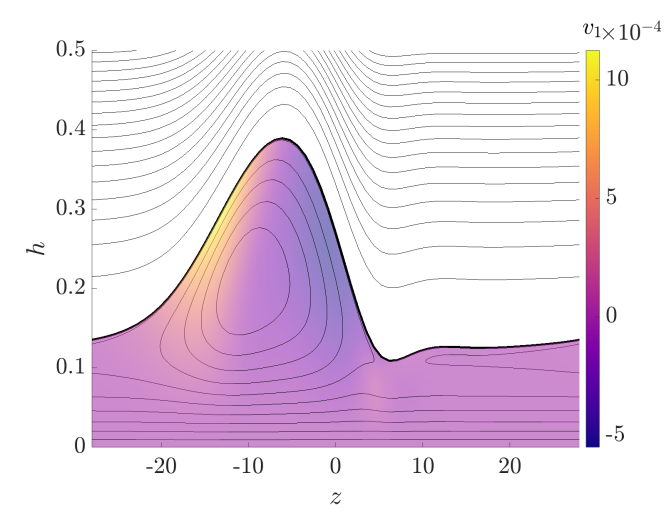

(a) Insoluble, $\bar{\Gamma}=0.5$

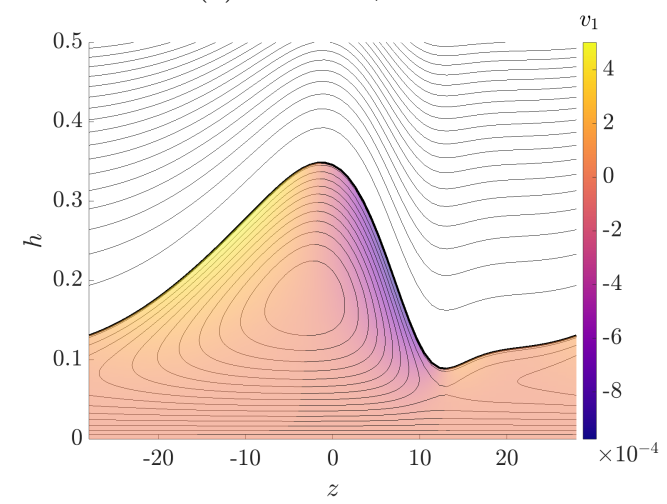

(c) Insoluble, $\bar{\Gamma}=0.75$

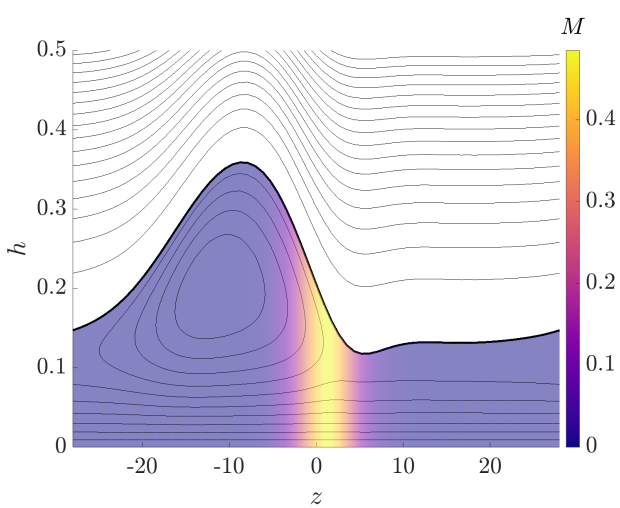

(b) Soluble, $\bar{\Gamma}=0.5$

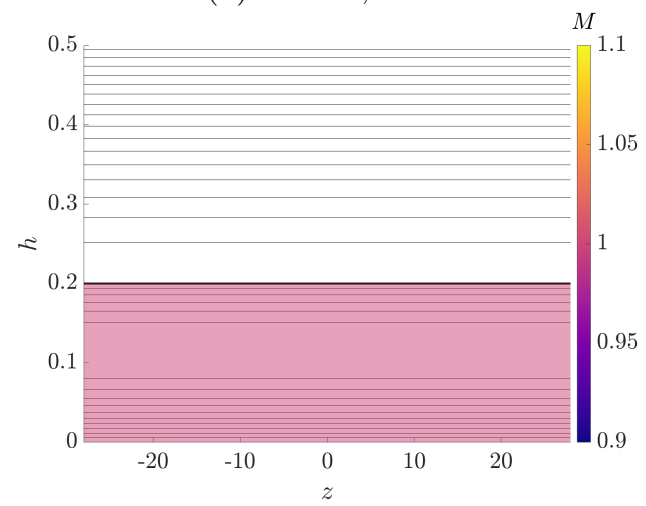

(d) Soluble, $\bar{\Gamma}=0.75$

Figure 6. Interfacial deformation (thick black line) for the system with $(\mathrm{a}, \mathrm{c})$ insoluble or $(\mathrm{b}, \mathrm{d})$ soluble surfactant and for two different values of $\bar{\Gamma}$. The thin black lines shown within the fluids are the streamlines which are equally spaced in $\Psi_{1}, \Psi_{2}$, and the colours in each figure correspond to $(\mathrm{a}, \mathrm{c})$ the vertical velocity $v_{1}$, or $(\mathrm{b}, \mathrm{d})$ the micelle concentration $M$. The parameter values used are $h_{0}=0.2$ and $m=0.5$. The rest of the parameters are the same as in figure $2 \mathrm{~b}$ and $\epsilon=0.1$. The time evolution of the interfacial wave and interfacial surfactant concentration for cases (c) and (d) can be seen in Videos 6-7 provided as supplementary material.

an unstable density stratification by assuming a larger upper fluid density compared to that of the lower fluid, so that the density ratio is larger than 1 . In particular, we take $(1-r) B o=-20 \epsilon^{2}=-0.2$ (cf. (2.35) ) and keep the rest of the parameters the same as in the calculation presented in figure $6 \mathrm{~d}$ (note that the flow dynamics is affected by the factor $(1-r)$ Bo through $f_{x}$, see 2.18$)$, but does not depend on the two components $(1-r)$ and $B o$ individually). The value of the above density stratification term is chosen such that gravity effects overcome the stabilising influence of micellisation and so that the flow is unstable (in fact, the critical value below which the system is unstable is found to be $(1-r) B o=-0.15)$. We note that even though the flow in this case a susceptible to the Rayleigh-Taylor instability, the interface saturates to a stable structure due to the presence of shear (Babchin et al. 1983).

The final saturated interfacial wave can be seen in figure $8 \mathrm{a}$, whereas the time evolution of the solution norm and travelling wave speed $c$ can be found in figure $8 \mathrm{~b}$. Even though the undisturbed thickness of the lower fluid occupies $1 / 5$ of the channel height (since $\left.h_{0}=0.2\right)$, the shape of the interface develops into a solitary pulse that is seen to be as tall as $2 / 3$ of the channel. The streamlines reveal once more the presence of an eddy 

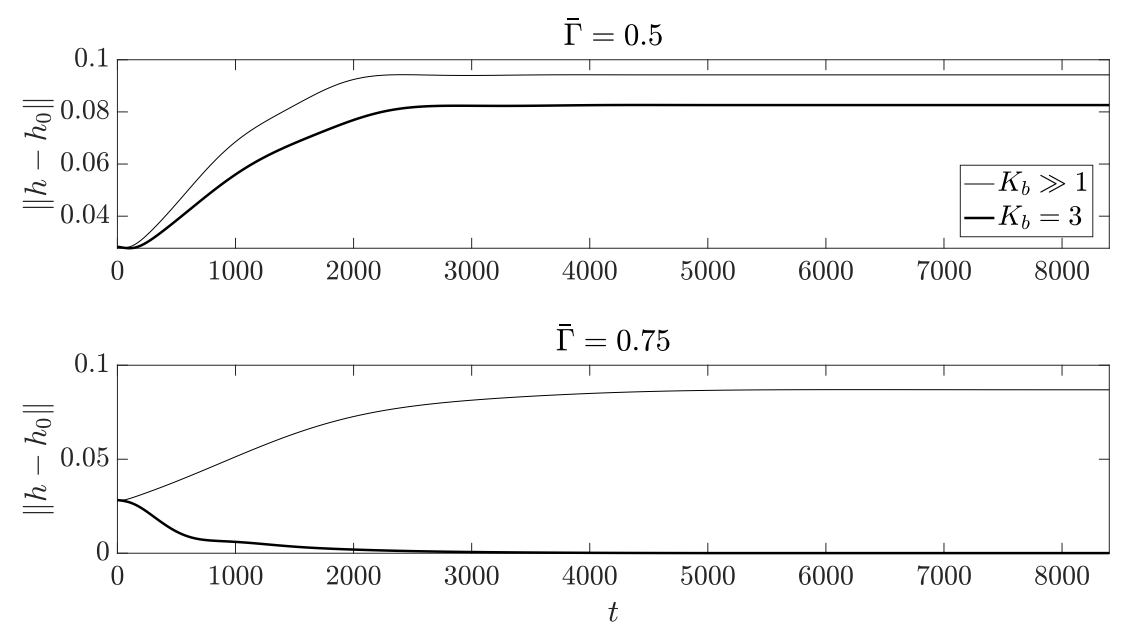

FiguRE 7. Norms of interfacial displacement for different values of solubility parameter $K_{b}$, corresponding to the simulations presented in figure 6 .

but this time it is trapped in the main part of the solitary pulse. In fact, a second recirculation zone exists in the upper fluid, located at about the same height as the pulse and following a clockwise rotation in the whole domain range (the flow in the frame of reference moving with wave speed $c$ is from left to right above the two re-circulation regions and from right to left below them). This means that a substantial portion of the upper fluid is transported along with the pulse. The existence of two stagnation points on the interface can be also seen at around $x=5$ and $x=13$ (depicted with black dot markers in figure 8 8 ), indicating locations where the direction of rotation changes.

A large concentration of micelles is formed under the pulse, illustrated with colour in figure 8 a. The saturated concentration of monomers at the interface and in the bulk, together with the corresponding interface-to-bulk flux $J_{b 0}=B i\left(K_{b} C_{0}(1-\Gamma)-\Gamma\right)$ are illustrated in figure $8 \mathrm{c}$ with dashed, dotted and solid lines, respectively. The concentrations are seen to be constant in approximately half of the domain but in the vicinity of the pulse large variations are observed.

Finally, we note that the solitary wave devoid of surfactant (i.e. the solution obtained from a numerical computation for the clean problem with constant surface tension) exhibits a larger saturated amplitude, with a second eddy residing within the upper fluid to the right of the pulse. The corresponding pulse with insoluble surfactant does not have a fixed from but its shape changes periodically in time. These remarks suggest that the presence of micelles have a stabilising influence on the flow, either through reducing the pulse amplitude or regularising the time-periodicity observed in the insoluble surfactant case.

\subsection{Travelling waves}

The results demonstrated in section 3.3 provide evidence of the existence of saturated travelling waves as solutions of the problem studied here. It is interesting to investigate these travelling wave solutions in a greater detail by examining the bifurcations from which they emerge and their properties as various geometrical or physical parameters are varied (such as the domain length or the fluid thicknesses). This can be achieved by using the continuation and bifurcation software AUTO-07p (Doedel \& Oldman 2009), which employs Newton's method and follows the solutions in parameter space. 


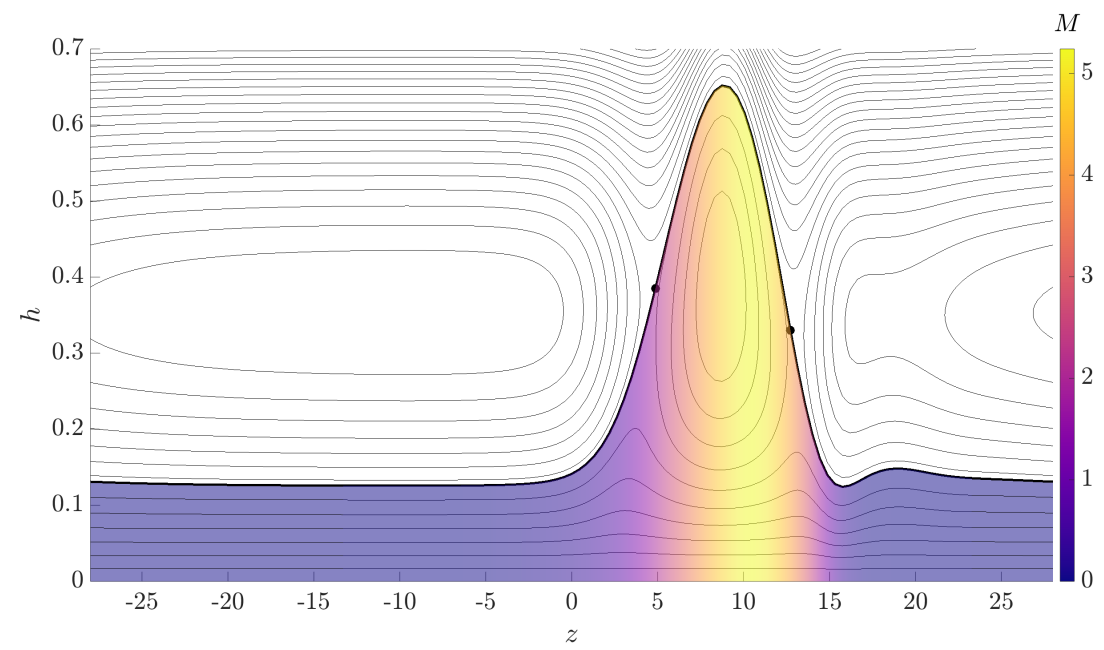

(a)

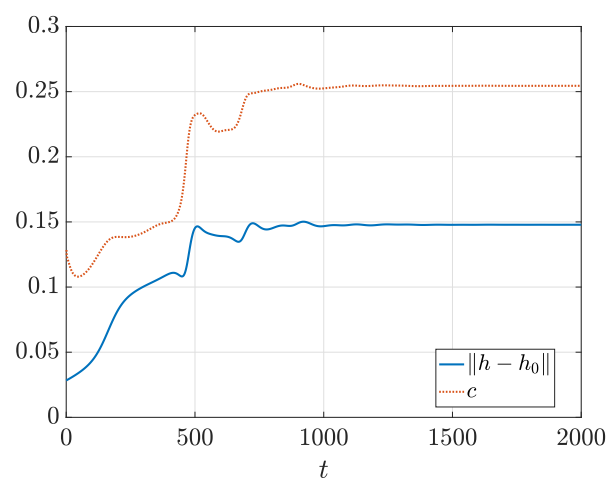

(b)

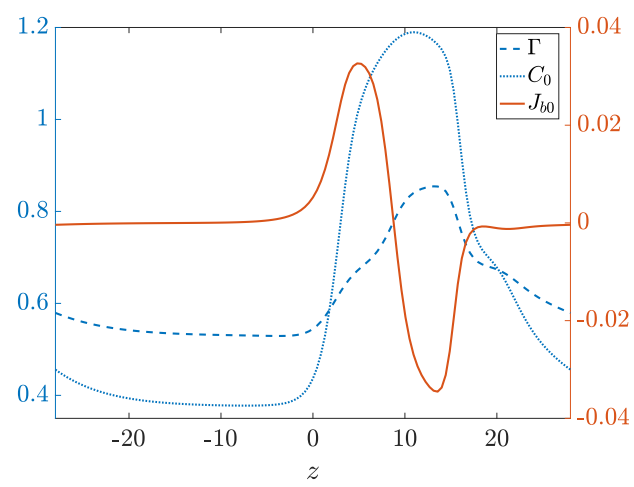

(c)

FIgURE 8. Results for section 3.3.4 corresponding to concentrations above the CMC and unstable density stratification, imposed by using $(1-r) B o=-0.2$ (the rest of the parameters are the same as in figure 6d). (a) Interfacial deformation (thick black line), streamlines in the two fluids (thin black lines), which are equally spaced in $\Psi_{1}, \Psi_{2}$, and micelle concentration (colour). The two black dots denote stagnation points. (b) Norm of interfacial displacement (solid blue line) and travelling wave speed (dotted red line). (c) Interfacial surfactant concentration (dashed blue line), monomer concentration in the bulk (dotted blue line) and interface-to-bulk flux $J_{b 0}$ (solid red line, right-hand-side $y$-axis).

Travelling wave solutions are sought directly by working in the travelling wave frame $z=x-c t$, where $c$ is the a priori unknown wave speed, in which case the system of equations 2.32, 2.33), 2.30 is transformed into a boundary-value problem. The resulting nonlinear system of ODEs is then solved using an initial guess constructed from linear stability theory, in order to latch onto the bifurcation branch that emanates from the neutral stability point where the linearised growth rate $s=0$ - see Appendix A for more details. Continuation is performed in terms of the travelling-wave speed $c$ and one other parameter, here chosen to be either the (half) domain length $L$ or the lower fluid thickness $h_{0}$, whereas the rest of the parameters are kept fixed. Figure 9 demonstrates the resulting interfacial waves for varying $L$ (panel (a)) or varying $h_{0}$ (panel (b)) and for fixed $K_{b}=10$ (the remaining parameters are the same as in figure 3 ). In the former case, 


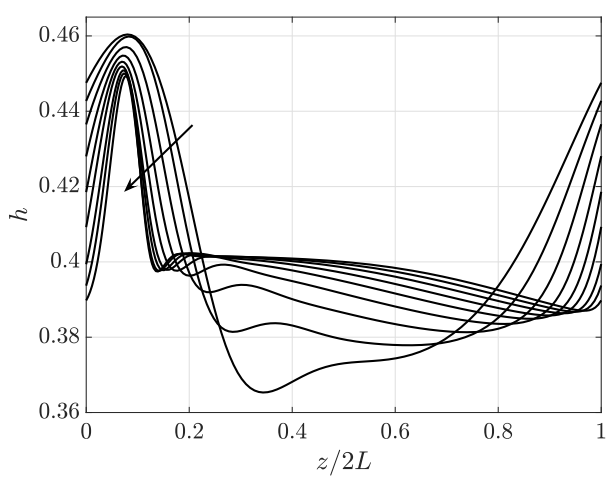

(a)

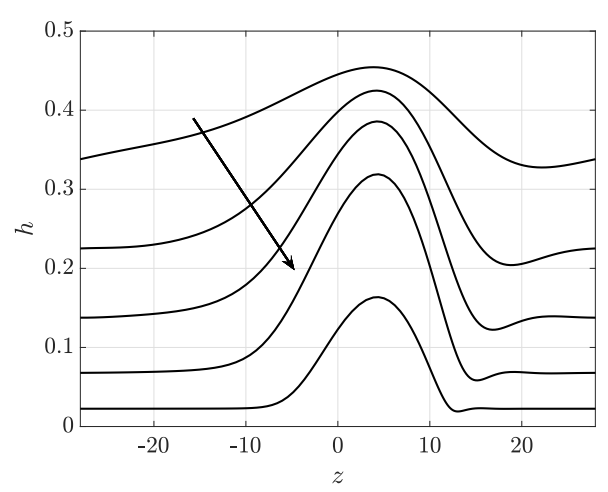

(b)

FiguRE 9. Interfacial profiles for (a) varying $L$ and fixed $h_{0}=0.4$, and (b) varying $h_{0}$ and fixed $L=28$. The arrows show the direction of increasing $L$ or decreasing $h_{0}$ in each panel, respectively. The depicted solutions are obtained for $K_{b}=10$ and the rest of the parameters are the same as in figure 3

$h_{0}=0.4$ is fixed and $L$ increases from $L=28$ (as used in the results of section 3.3) up to $L=200$. The interface deflection is shown in figure $9 \mathrm{a}$ in a scaled horizontal domain $[0,1]$ for nine different values of $L$ varying from $L=40$ (curve with lowest trough) to $L=200$ (curve with thinnest pulse). The increase of the domain length causes the crest of the wave to become thinner and slightly shorter, and makes the structure more localised in the sense that it becomes flatter between the peaks. The variation of the corresponding travelling wave speed against $L$ is shown in figure $10 \mathrm{a}$ and is seen increase monotonically as $L$ becomes larger. In the second continuation case, the domain length is fixed to $L=28$ and the lower fluid thickness decreases from $h_{0}=0.4$ down to $h_{0}=0.05$ - figure $9 \mathrm{p}$ illustrates a series of interfacial waves varying from $h_{0}=0.38$ (top curve) to $h_{0}=0.05$ (bottom curve). The reduction in $h_{0}$ leads to the steepening of the interface around the wave crest and the flattening of the rest of the interface - for sufficiently small values of $h_{0}$, the wave is seen to become a solitary-type pulse followed by a small depression. The surfactant is found to accumulate in the vicinity of the depression, both at the interface and in the bulk fluid (results not shown). Also, the wave speed $c$ diminishes to zero as $h_{0}$ is decreased (figure $10 \mathrm{p}$ ). We note that carrying out time-dependent computations of the lubrication model for the cases with relatively small $h_{0}$ requires much larger final times and smaller resolutions until a saturated wave is attained, which highlights one of the main advantages of the continuation method. The existence of solitary waves at the interface between two viscous fluids in a channel has been reported before by Power et al. (1991); Samanta (2013), and they have been observed in Couette-flow experiments by Gallagher et al. (1996).

The effect of surfactant solubility on the interfacial travelling waves can be investigated by performing continuation with respect to the solubility parameter $K_{b}$. This is achieved by considering the solutions obtained for $L=28, h_{0}=0.4, K_{b}=10$ and decreasing $K_{b}$, which is found to reduce the wave amplitude until around $K_{b}^{c}=2.62$ that signifies a bifurcation point; below this value the system is stabilised and the interface is flat. This is in line with the results presented in figures 3 and 43 . We end this section with a comparison between the solution obtained at $L=200, h_{0}=0.4, K_{b}=10$ (cf. figure $9 \mathrm{a}$ ) and the corresponding solution found using the insoluble system, which is depicted in figure 11. The interface deformation (top panel) and interfacial surfactant concentration (bottom panel) are shown with solid and dotted lines for the soluble and 


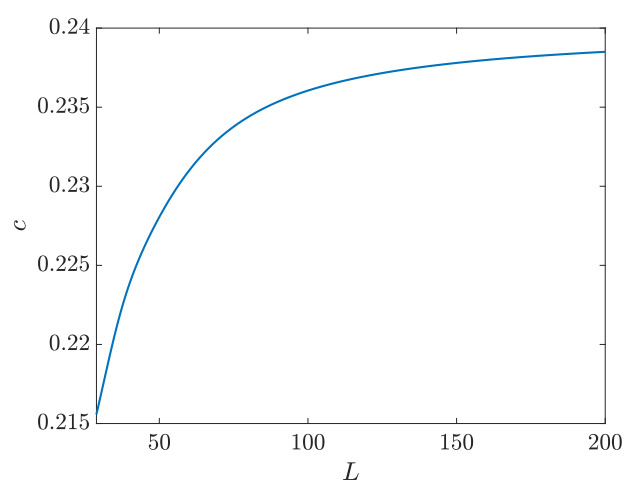

(a)

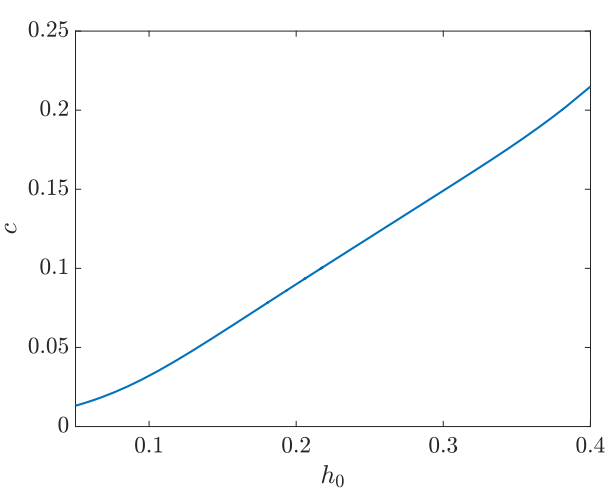

(b)

FiguRE 10. Travelling wave speeds for (a) varying $L$ and fixed $h_{0}=0.4$, and (b) varying $h_{0}$ and fixed $L=28$. The depicted solutions are obtained for $K_{b}=10$ and the rest of the parameters are the same as in figure 3 .

insoluble cases, respectively. As already demonstrated by previous results in this work, the strengthening of surfactant solubility diminishes the wave amplitude but also leads to the wave becoming less localised. Moreover, the distribution of surfactant concentration at the interface is seen to be reduced in amplitude and confined in a smaller region in the soluble case - this is expected due to the desorption kinetics towards the bulk fluid. The behaviour of the surfactant concentration which shows significant variation throughout the channel in contrast to the localised interfacial structure has been observed before by Thompson \& Blyth (2016) in their study of three-layer free-surface flows.

\section{4. (In)stability mechanisms}

The primary aim of this section is to investigate the physical reasons that cause the system to become unstable for specific fluid properties and explain the effect of surfactant solubility on the stability. In particular, we aim to understand why the factor $\left(m-n^{2}\right)$ is important and what makes the problem to change its stability behaviour when this boundary is crossed in parameter space. A creeping two-layer flow in a channel devoid of surfactant is known to be stable when the density is uniform (Yih 1967), but when a dilute concentration of surfactant is added, then the insoluble limit is appropriate and it has been found that the interface is unstable if $m<n^{2}$, and stable otherwise (Frenkel \& Halpern 2002; Halpern \& Frenkel 2003, Wei 2005). The impact of surfactant solubility on these stability properties will be examined here.

The (in)stability mechanisms will be investigated by obtaining the linear equations of motion, which is achieved by writing $h(x, t)=h_{0}+\delta \hat{h}(x, t), \Gamma(x, t)=\bar{\Gamma}+\delta \hat{\Gamma}(x, t)$, etc. for $\delta \ll 1$, and keeping only linear terms in the lubrication model. The following linear equation for the interfacial deformation is hence obtained from 2.32

$$
\hat{h}_{t}+\alpha_{1} \hat{h}_{x}-\beta_{1} \hat{h}_{x x}+\gamma_{1} \hat{h}_{x x x x}+\delta_{1} \hat{\Gamma}_{x x}=0,
$$

where the coefficients $\alpha_{1}, \beta_{1}, \gamma_{1}, \delta_{1}$ are functions of $n, m, r$ and other non-dimensional parameters and are given in Appendix B. The full linearised system is also provided in the same Appendix. Coefficient $\beta_{1}$ depends on the factor $(1-r)$ and hence represents the influence of density stratification (it can become negative in cases of unstable density stratification); term $\gamma_{1}$ corresponds to surface tension effects and specifically depends on the undisturbed value $\bar{\gamma}=1+\beta_{s} \ln (1-\bar{\Gamma})$; coefficient $\delta_{1}$ introduces the effect of 

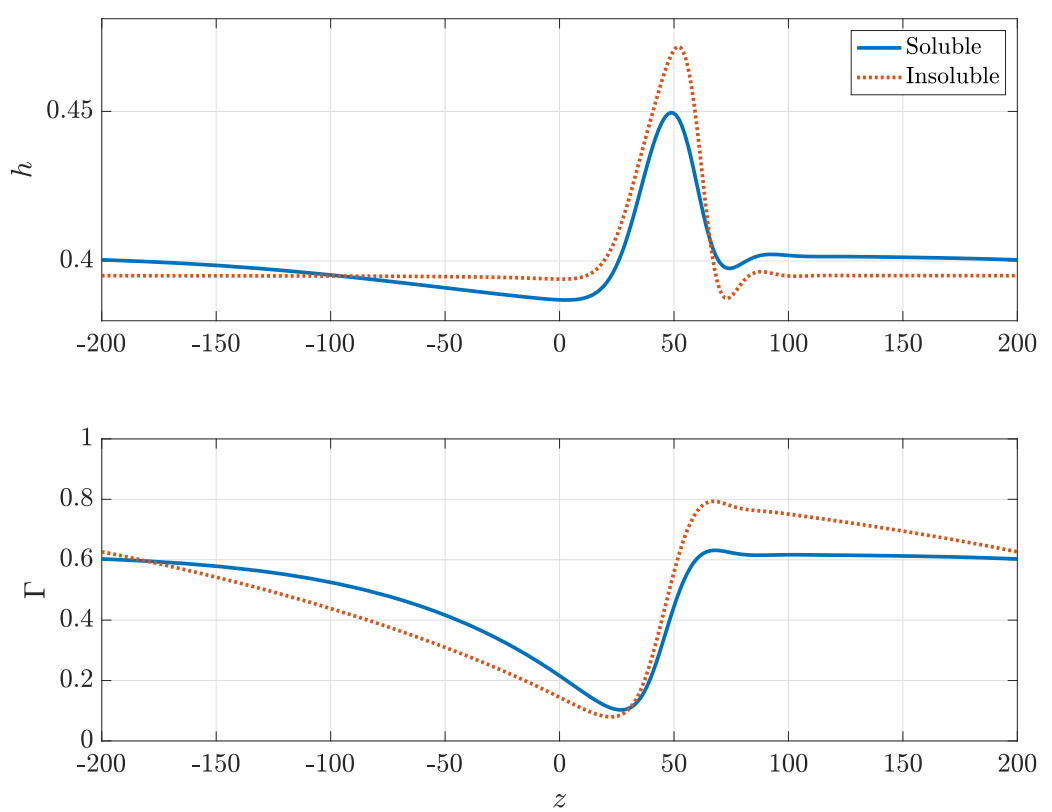

FiguRE 11. Interfacial profiles (top) and surfactant concentrations at the interface (bottom) obtained for $L=200, h_{0}=0.4, K_{b}=10$, and the rest of the parameters are the same as in figure 3. Solid lines demonstrate solutions of the full system with soluble surfactant and dotted lines are used for solutions of the corresponding insoluble system.

Marangoni forces into the dynamics and is proportional to the crucial factor $\left(m-n^{2}\right)$. Clearly the sign of $\left(m-n^{2}\right)$ in the coefficient of the $\Gamma_{x x}$ term affects the way Marangoni forces are acting on the interface (Wei 2005).

Having obtained equation (4.1), we can perform an analysis similar to that for energy budgets (Picardo et al. 2016) by multiplying the equation with $\hat{h}$ and integrating over one spatial period. The "energy" equation reads

$$
\frac{\mathrm{d}}{\mathrm{d} t} \int_{-L}^{L} \frac{1}{2} \hat{h}^{2} \mathrm{~d} x=-\beta_{1} \int_{-L}^{L} \hat{h}_{x}^{2} \mathrm{~d} x-\gamma_{1} \int_{-L}^{L} \hat{h}_{x x}^{2} \mathrm{~d} x+\delta_{1} \int_{-L}^{L} \hat{h}_{x} \hat{\Gamma}_{x} \mathrm{~d} x,
$$

where the left-hand-side term represents the rate of change of the interfacial disturbance. In a stably stratified system, the first two terms on the right-hand-side are negative and hence always stabilising due to the action of gravity or surface tension, respectively. The third integral on the right-hand-side is found to be negative in numerical computations of the system with insoluble surfactant, hence it has a destabilising influence when $\delta_{1}<0$ that is when $m<n^{2}$, otherwise the systems is stable; this is in line with the known result from the literature on the stability of two-layer channel flow with insoluble surfactants (Frenkel \& Halpern 2002; Halpern \& Frenkel 2003).

Next, we write the eigenfunctions $\hat{h}, \hat{\Gamma}$ in a normal-mode form $\hat{h}(x, t)=\tilde{h} \mathrm{e}^{i k x+\sigma t}+$ c.c., $\hat{\Gamma}(x, t)=\tilde{\Gamma} \mathrm{e}^{i k x+\sigma t}+$ c.c., where c.c. denotes the complex conjugate. The amplitude of the interface disturbance is normalised by setting $\tilde{h}=1$ and the concentration disturbance amplitude is written as $\tilde{\Gamma}=G \mathrm{e}^{i \phi}$, where $\phi$ is the phase difference between the interface deformation and concentration waves, and $G>0$. Applying these normal modes, we 

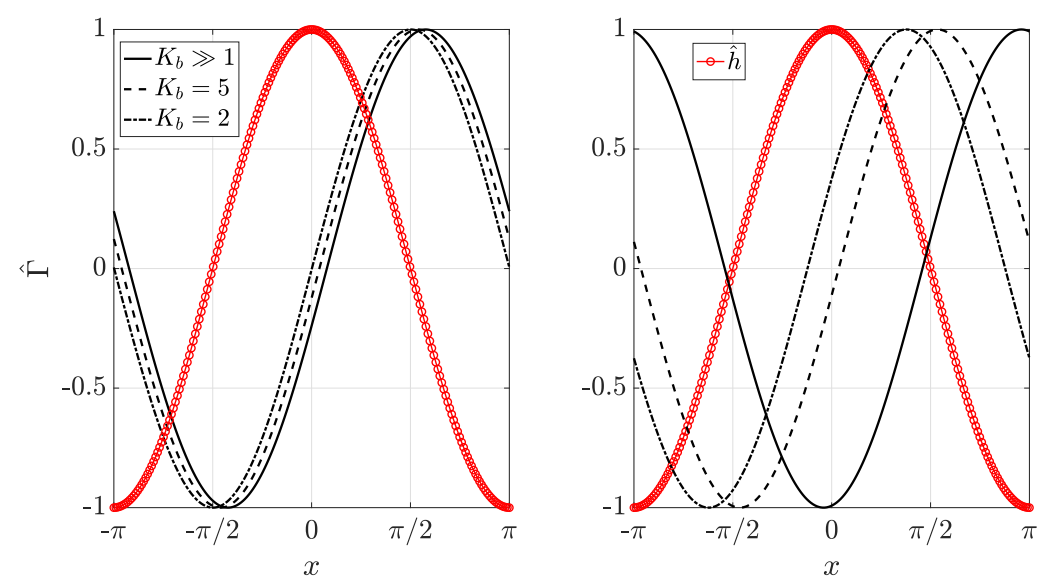

FiguRE 12. Wave forms of the normalised eigenfunctions for the interface deformation (red) and interfacial surfactant concentration (black). The results are rescaled in the domain $x \in[-\pi, \pi]$ for easier realisation of the phases. The three black curves in each panel correspond to different values of solubility parameter $K_{b}$ as quoted in the legend. The left panel uses the same parameter values as figure 3 satisfying $m<n^{2}$, while the right panel corresponds to figure 5 with $m \geqslant n^{2}$.

obtain from 4.1 on taking the real part,

$$
\Re(\sigma)=-\beta_{1} k^{2}-\gamma_{1} k^{4}+\delta_{1} k^{2} G \cos \phi .
$$

Assuming that the system is stably stratified in which case the first term on the righthand-side is negative, then the condition for instability, given by $\Re(\sigma)>0$, can be satisfied only when the term $\delta_{1} \cos \phi$ is positive. If $m<n^{2}$ in which case $\delta_{1}<0$, then instability is possible when $\cos \phi<0$ or $\phi$ in the range $(\pi / 2,3 \pi / 2)$. On the other hand, for $m \geqslant n^{2}$ or equivalently $\delta_{1}>0$, instability is only possible when $\cos \phi>0$ or $\phi \in(-\pi / 2, \pi / 2)$.

Figure 12 demonstrates the normalised perturbation eigenfunctions corresponding to the dominant mode for the interface deformation $\hat{h}$ (red lines with circles) and interfacial surfactant concentration $\hat{\Gamma}$ (black lines). The concentration wave forms are presented separately for $m<n^{2}$ (left) and $m \geqslant n^{2}$ (right) and in each case they are shown for three different values of the solubility parameter $K_{b}$ - in fact, the parameter sets used in the two panels correspond to the cases presented earlier in figures 3 and 5 , respectively. It can be seen that for $m<n^{2}$, the phase difference between $\hat{h}$ and $\hat{\Gamma}$ in the case of insoluble surfactant $\left(K_{b} \gg 1\right.$, solid line) is bigger than $\pi / 2$, confirming that the system is unstable according to the discussion in the previous paragraph. When surfactant solubility is imposed (equivalently, as $K_{b}$ is reduced), the concentration wave shifts to the left and eventually the phase becomes equal to $\pi / 2$ for small enough $K_{b}$ (dash-dot line). This behaviour leads to stability as already expected from the results of section 3.3.1. When $m \geqslant n^{2}$, the phase corresponding to the insoluble system is approximately $\pi$ (solid line), hence the system is stable as this is greater than $\pi / 2$ and $\cos \phi<0$. Solubility effects lead to the shifting of the concentration wave to a phase that is smaller than $\pi / 2$ (see dashed and dash-dot lines) and hence to stability.

Once the phase difference between the interface deformation and concentration is known, then the physical mechanism leading to instability is connected with the action of Marangoni forces at the interface. This has been discussed extensively in related literature for insoluble surfactant, see the studies by Frenkel \& Halpern (2002); Blyth \& Pozrikidis (2004); Wei (2005). In short, local accumulation of surfactant establishes surface tension 
gradients that, by virtue of the phase difference, tend to drive fluid toward the troughs and peaks of the interfacial deformation wave, forcing it to grow in amplitude.

\section{Conclusions}

The nonlinear stability of long waves at the interface between two viscous fluids in a channel has been investigated numerically, in the case when one of the fluids is contaminated with a surfactant. In particular, the effect of surfactant solubility on the interfacial stability has been assessed, including cases where the surfactant concentration exceeds the critical micelle concentration. In such situations, micelles are formed in the bulk fluid and this affects the dynamical behaviour of the surface tension that varies in response to the local interfacial concentration. A complete set of governing equations for the hydrodynamics and surfactant transport has been presented and then reduced using lubrication theory. The simplified set of equations and boundary conditions has been solved to derive a strongly nonlinear system of evolution equations; this system comprises four partial differential equations describing the motion of the interface, the variation of interfacial concentration of surfactant, and the corresponding leadingorder bulk and micelle concentrations. The two evolution equations for the surfactant concentrations in the bulk fluid (for monomers and micelles) have been obtained by assuming rapid vertical diffusion and by averaging the flow velocities across the fluid. The derived long-wave model is valid for inertialess flows but includes other pertinent physical effects such as density and viscosity stratification, shear, and surfactant kinetics due to adsorption/desorption or micelle formation/breakup.

When a two-layer, stably stratified shear flow in a straight channel is devoid of surfactant, instability can only arise when inertia is present (Yih 1967), but the addition of insoluble surfactant at the interface can introduce unstable modes even in the inertialess case (Halpern \& Frenkel 2003, Blyth \& Pozrikidis 2004) if certain fluid properties are satisfied (namely if the fluid viscosity ratio $m$ and thickness ratio $n$ satisfy the condition $m<n^{2}$ ). When the surfactant is soluble as in the problem studied here, the onset of instability has been previously investigated in Kalogirou \& Blyth (2019) for arbitrary wavenumbers by linearising the full system of governing equations and applying normalmode analysis. A similar linear stability analysis of the long-wave model has been performed in this work and the obtained growth rates have been found to coincide with the results of Kalogirou \& Blyth (2019) for sufficiently small wavenumbers.

The established instabilities have been followed into the nonlinear regime and the dynamical behaviour of the system has been explored by undertaking a range of numerical computations for different values of the fluid viscosity ratio $m$, the thickness ratio $n$, and the surfactant solubility parameter $K_{b}$. The numerical results have demonstrated the existence of saturated travelling waves as solutions and the focus of the numerical investigation has been placed on identifying the effect of surfactant solubility on such travelling wave solutions. For $m<n^{2}$, it has been found that as the surfactant solubility effects are enhanced, the saturated interfacial waves attain smaller amplitudes; when the surfactant is sufficiently soluble, the interface returns to the flat undisturbed state at large times. The opposite phenomenon has been observed for $m \geqslant n^{2}$, in which case a sufficient amount of surfactant solubility can destabilise the interface (which remains flat if the surfactant is nearly insoluble) and give rise to coherent wave structures. In cases where the surfactant in the bulk reaches an average concentration that is beyond the $\mathrm{CMC}$, the inertialess flow has been found to be stable. Finally, such stable surfactantladen flows have been investigated under the effect of density stratification. Adverse density stratification can destabilise the flow if the density ratio exceeds a critical value 
in which case a large-amplitude saturated pulse of a solitary-wave type has been seen to emerge at the interface; however, the micelles that are formed still have a stabilising influence in the sense of reducing the pulse amplitude compared to that obtained in the clean case.

Travelling wave solutions have been also constructed directly by solving a boundaryvalue problem that included the unknown wave speed $c$. The method of continuation has been used to track how the interfacial waves and their speed change as the domain length $L$ or undisturbed lower fluid thickness $h_{0}$ vary. The results have indicated that both the increase of the domain length $L$ or the decrease of the thickness $h_{0}$ lead to a more localised structure. In fact, the interface becomes a solitary pulse when $h_{0}$ is sufficiently small. The strengthening of surfactant solubility (equivalently the reduction of the value of parameter $K_{b}$ ) has the opposite effect, i.e. it makes the wave less localised, and also causes the wave amplitude to shrink. The distribution of surfactant at the interface is also reduced in amplitude when the surfactant is soluble, and it demonstrates a smaller variation along the domain.

The mechanism responsible for interfacial (in)stability has been explained in terms of the phase difference between the perturbation eigenfunctions for the interface deformation $\hat{h}$ and interfacial surfactant concentration $\hat{\Gamma}$ (for the dominant mode). This has been achieved by writing a linearised interfacial evolution equation and obtaining the growth rate - this growth rate, and consequently the stability of the interface, has been found to be crucially dependent on the factor $\left(m-n^{2}\right)$, as already realised in related studies for insoluble surfactant (Frenkel \& Halpern 2002; Halpern \& Frenkel 2003, Blyth \& Pozrikidis 2004; Wei 2005). It has been demonstrated that the effect of surfactant solubility is to modify the phase difference $\phi$ between the interface deformation and concentration waves. For $m<n^{2}$, the phase changes from a value $\phi>\pi / 2$ found for insoluble surfactant to $\phi=\pi / 2$ when the solubility is strong enough, which stabilises the flow. On the other hand, for $m \geqslant n^{2}$, a sufficient amount of solubility is able to shift the phase difference to a value $\phi<\pi / 2$ and make the flow unstable. The stability behaviour of the system based on the above remarks is in line with the nonlinear numerical solutions presented in this work, as well as results from linear stability analysis (Kalogirou \& Blyth 2019 ).

The formulation of the model presented in this work includes interfacial elasticity (or equivalently surface tension gradients due to variation of surfactant concentration) and neglects the effects of interfacial viscosity or rheology. Surfactant absorbed at fluid-fluid interfaces might potentially also give rise to shear and dilatational surface viscosities (Langevin 2014). Typical systems like the one considered in this study have been investigated experimentally using the soluble surfactant SDS (Georgantaki et al. 2016); this type of surfactant has been analysed in a number of studies in the literature (Gupta \& Wasan 1974, Shen et al. 2002, Zell et al. 2014) that reported SDS measurements (both above and below CMC) demonstrating a surface viscosity that is immeasurably small. Nevertheless, interfacial viscosity may play an important role in many practical scenarios using different surfactants (see, for example, the study by Ponce-Torres et al. (2017) on the break-up of surfactant-laden droplets). The predominant influence of surface viscosity is to weaken Marangoni forces, and hence to ameliorate Marangoni-driven instability, and as such it would be an interesting effect to include in a future extension to the present work.

This study represents the first attempt to investigate the nonlinear dynamics of interfaces in inertialess multi-layer flows with surfactant above the CMC. Extending the current work and the long-wave model to include inertia can provide a useful framework for examining the competition between the interacting effects of micellisation 
and inertia. Finally, the range of validity of the lubrication model can be estimated by direct comparisons with direct numerical simulations of the full system of governing equations. Both of these open questions are subjects of ongoing investigations.

\section{Acknowledgements}

A. Kalogirou acknowledges funding by the Leverhulme Trust through an Early Career Fellowship.

\section{Appendix A. Implementation in AUTO-07p}

The system of equations 2.32 , 2.33), 2.30 is rescaled in the domain $[0,1]$ by introducing a scaled variable $X=z /(2 L)$ and written as a system of ODEs of the form

$$
\dot{\boldsymbol{U}}=2 L \mathcal{A}(\boldsymbol{U})
$$

where the dot denotes differentiation with respect to $X$,

$$
\boldsymbol{U}=\left(U_{1}, U_{2}, U_{3}, U_{4}, U_{5}, U_{6}, U_{7}, U_{8}, U_{9}, U_{10}\right)=\left(h, h_{z}, h_{z z}, h_{z z z}, \Gamma, \Gamma_{z}, C_{0}, C_{0 z}, M_{0}, M_{0 z}\right),
$$

is the vector of unknowns and the right-hand-side vector is defined by

$$
\mathcal{A}(\boldsymbol{U})=\left(U_{2}, U_{3}, U_{4}, F_{h}(\boldsymbol{U}), U_{6}, F_{\Gamma}(\boldsymbol{U}), U_{8}, F_{C}(\boldsymbol{U}), U_{10}, F_{M}(\boldsymbol{U})\right) .
$$

The functions $F_{h}(\boldsymbol{U}), F_{\Gamma}(\boldsymbol{U}), F_{C}(\boldsymbol{U}), F_{M}(\boldsymbol{U})$ are found by re-arranging the respective equations from (2.32), 2.33, 2.30 in terms of $h_{z z z z}, \Gamma_{z z}, C_{0 z z}, M_{0 z z}$.

The boundary value problem is completed by introducing appropriate boundary and integral conditions. In particular, six periodic boundary conditions are imposed for variables $h, h_{z}, h_{z z}, \Gamma, C_{0}, M_{0}$ through

$$
U_{i}(0)=U_{i}(1), \quad i=1,2,3,5,7,9 .
$$

Note that periodicity conditions on $h_{z z z}, \Gamma_{z}, C_{0 z}, M_{0 z}$ are not imposed; in fact, equation (2.32 written in a steady travelling wave frame, can be integrated once in $z$ making such condition on $h_{z z z}$ unnecessary. The corresponding conditions on $\Gamma_{z}, C_{0 z}, M_{0 z}$ are also redundant in view of integral constraints (iv) and (v) below. In addition to the boundary conditions A 4 , five integral conditions are imposed as follows:

(i) a phase condition that prevents the continuation algorithm from detecting translationally-invariant solutions, given in the form $\int_{0}^{1} U_{1} U_{1}^{\prime} \mathrm{d} X=0$, where the prime denotes the derivative along the solution curve in parameter space.

(ii) the volume of the fluid is conserved, i.e. the integral of $h=U_{1}$ is constant, given by $\int_{0}^{1} U_{1} \mathrm{~d} X=h_{0}$.

(iii) the total mass of surfactant is conserved, namely $\int_{0}^{1}\left(\frac{1}{\beta_{b}}\left(U_{7}+U_{9}\right) U_{1}+U_{5}\right) \mathrm{d} X=$ $\mathcal{M}_{0}$, where $\mathcal{M}_{0}$ is the initial mass calculated from 3.4 .

(iv) the integral of $J_{b 0}$ is zero, $\int_{0}^{1} J_{b 0}\left(U_{5}, U_{7}\right) \mathrm{d} X=0$. This condition is obtained by integrating equation 2.33 (written in a travelling wave frame-of-reference) and assuming that periodicity on $U_{6}=\Gamma_{z}$ is satisfied - it is therefore equivalent to including such a periodicity condition in A4, which is currently omitted.

(v) the integral of $h J_{m 0}$ is zero, i.e. $\int_{0}^{1} U_{1} J_{m 0}\left(U_{7}, U_{9}\right) \mathrm{d} X=0$. This condition is obtained by combining the equations 2.30 and 2.32 and integrating the result. As with the previous integral condition, this constraint is associated with the requirement of periodicity for $U_{8}=C_{0 z}, U_{10}=M_{0 z}$. 
Therefore we have a system of size $N_{D I M}=10$ solved with $N_{B C}=6$ boundary conditions and $N_{I N T}=5$ integral conditions. The number of free parameters in the continuation is determined by the relation $N_{C O N T}=N_{B C}+N_{I N T}-N_{D I M}+1=2$, so there are two free parameters in this case. We perform continuation in terms of the travelling-wave speed $c$ and one other geometrical or physical parameter, such as the (half) domain length $L$ or the lower fluid thickness $h_{0}$, while the rest of the parameters are kept fixed.

Finally, the initial conditions are at a bifurcation point where $\Re(\sigma)=0$. We find the critical wavenumber for instability $k_{c}$ and set $L=\pi / k_{c}$, while the wave speed $c$ is set to $c=-\Im(\sigma) / k_{c}$. The initial condition for $h$ is then taken to be a perturbation of the steady state of the form $h(X, 0)=h_{0}+\delta \cos (2 \pi X)$, with small-amplitude coefficient $\delta=0.001$, while the initial conditions for the remaining parameters $\Gamma, C_{0}, M_{0}$ are such that to satisfy the linear system of governing equations.

\section{Appendix B. Linearised lubrication model}

Small perturbations are introduced to the steady state by $h(x, t)=h_{0}+\delta \hat{h}(x, t)$, $\Gamma(x, t)=\bar{\Gamma}+\delta \hat{\Gamma}(x, t)$, etc $(\delta \ll 1)$, and only linear terms are kept in the lubrication model (given below in the case of $C<C_{C M C}$ ), giving

$$
\begin{aligned}
& \hat{h}_{t}+\alpha_{1} \hat{h}_{x}-\beta_{1} \hat{h}_{x x}+\gamma_{1} \hat{h}_{x x x x}+\delta_{1} \hat{\Gamma}_{x x}=0, \\
& \hat{\Gamma}_{t}+\alpha_{2} \hat{h}_{x}+\beta_{2} \hat{h}_{x x}-\gamma_{2} \hat{h}_{x x x x}-\delta_{2} \hat{\Gamma}_{x x}+\varepsilon_{2} \hat{\Gamma}_{x}-\hat{J}_{b 0}=0, \\
& \hat{C}_{0 t}+\alpha_{3} \hat{C}_{0 x}-\beta_{3} \hat{C}_{0 x x}+\gamma_{3} \hat{J}_{b 0}=0 .
\end{aligned}
$$

The coefficients that appear in the linearised system of equations (B 1) are given by

$$
\begin{aligned}
& \alpha_{1}=\frac{m\left(n^{4}+(2 m+2) n^{3}+(4 m+2) n^{2}+4 m n+m^{2}\right)}{(n+m) \Phi}, \\
& \alpha_{2}=\frac{m \Psi(n+1)^{2} \bar{\Gamma}}{(n+m) \Phi}, \quad \alpha_{3}=\frac{m}{2(n+m)}, \\
& \beta_{1}=\frac{(1-r) B o(n+m) n^{3}}{3 C a(n+1)^{3} \Phi}, \quad \beta_{2}=\frac{(1-r) B o\left(m-n^{2}\right) n^{2} \bar{\Gamma}}{2 C a(n+1)^{2} \Phi}, \quad \beta_{3}=\frac{1}{P e_{b}}, \\
& \gamma_{1}=\frac{\bar{\gamma}(n+m) n^{3}}{3 C a(n+1)^{3} \Phi}, \quad \gamma_{2}=\frac{\bar{\gamma}\left(m-n^{2}\right) n^{2} \bar{\Gamma}}{2 C a(n+1)^{2} \Phi}, \quad \gamma_{3}=\beta_{b}(n+1), \\
& \delta_{1}=\frac{M a\left(m-n^{2}\right) n^{2}}{2(1-\bar{\Gamma})(n+1)^{2} \Phi}, \quad(\mathrm{B} \\
& \delta_{2}=\frac{n^{5}+(4 m+1) n^{4}+10 m n^{3}+10 m n^{2}+m(m+4) n+m^{2}}{(n+1) P e_{s} \Phi}+\frac{n\left(n^{3}+m\right) M a \bar{\Gamma}}{(n+1)(1-\bar{\Gamma}) \Phi}, \\
& \varepsilon_{2}=\frac{m}{(n+m)},
\end{aligned}
$$

where

$$
\begin{aligned}
\Phi & =n^{4}+4 m n^{3}+6 m n^{2}+4 m n+m^{2}, \\
\Psi & =n^{3}+3 n^{2}+3 m n+m, \\
\bar{\gamma} & =1+\beta_{s} \ln (1-\bar{\Gamma}),
\end{aligned}
$$

and

$$
\hat{J}_{b 0}=B i\left(K_{b}(1-\bar{\Gamma}) \hat{C}_{0}-\left(1+K_{b} \bar{C}\right) \hat{\Gamma}\right)=\Delta_{3} \hat{C}_{0}-E_{3} \hat{\Gamma}
$$


Note that the above coefficients in $(\mathrm{B} 2)$ are always positive except from $\beta_{1}, \beta_{2}, \gamma_{2}, \delta_{1}$ which could also be negative (or even zero in the case of $\beta_{1}, \beta_{2}$ ). This can happen if the system is unstably stratified, in which case the term $(1-r)$ becomes negative, or due to a change in the sign of factor $\left(m-n^{2}\right)$ that depends on the fluid viscosity and thickness ratio.

\section{REFERENCES}

Afsar-Siddiqui, A. B., Luckham, P. F. \& Matar, O. 2003 The spreading of surfactant solutions on thin liquid films. Adv. Colloid Interfac. 106, 183-236.

Babchin, A. J., Frenkel, A. L., Levich, B. G. \& Sivashinsky, G. I. 1983 Nonlinear saturation of Rayleigh-Taylor instability in thin films. Phys. Fluids 26, 3159.

Barthelet, P., Charru, F. \& Fabre, J. 1995 Experimental study of interfacial long waves in a two-layer shear flow. J. Fluid Mech. 303, 23-53.

Bassom, A. P., Blyth, M. G. \& Papageorgiou, D. T. 2010 Nonlinear development of twolayer Couette-Poiseuille flow in the presence of surfactant. Phys. Fluids 22 (102102), 1-15.

BERRET, J.-C. 2006 Rheology of wormlike micelles: Equilibrium properties and shear banding transitions. In Molecular Gels: Materials with Self-Assembled Fibrillar Networks (ed. R. G. Weiss \& P. Terech), chap. 19, pp. 667-720. The Netherlands: Springer.

Blyth, M. G. \& Pozrikidis, C. 2004 Effect of surfactants on the stability of two-layer channel flow. J. Fluid Mech. 505, 59-86.

Blyth, M. G., Tseluiko, D., Lin, T.-S. \& Kalliadasis, S. 2018 Two-dimensional pulse dynamics and the formation of bound states on electrified falling films. J. Fluid Mech. $\mathbf{8 5 5}, 210-235$.

Breward, C. J. W. \& Howell, P. D. 2004 Straining flow of a micellar surfactant solution. Euro. J. Appl. Math. 15, 511-531.

Chang, C.-H. \& Franses, E. I. 1995 Adsorption dynamics of surfactants at the air/water interface: a critical review of mathematical models, data, and mechanisms. Colloid Surface A 100, 1-45.

Chen, L.-H. \& Lee, Y.-L. 2000 Adsorption behavior of surfactants and mass transfer in singledrop extraction. AIChE Journal 46, 160-168.

Craster, R. V., Matar, O. \& Papageorgiou, D. T. 2009 Breakup of surfactant-laden jets above the critical micelle concentration. J. Fluid Mech. 629, 195-219.

Danov, K. D., Vlahovska, P. M., Horozov, T., Dushskin, C. D., Kralchevsky, P. A., Menreteab, A. \& Broze, G. 1996 Adsorption from micellar surfactant solutions: Nonlinear theory and experiment. J. Colloid Interf. Sci. 183, 223-235.

Doedel, E. J. \& Oldman, B. E. 2009 AUTO-07P: Continuation and Bifurcation Software for Ordinary Differential Equations. Concordia University, available at http://cmvl.cs.concordia.ca/auto/.

Edmonstone, B. D., Craster, R. V. \& Matar, O. K. 2006 Surfactant-induced fingering phenomena beyond the critical micelle concentration. J. Fluid Mech. 564, 105-138.

Frenkel, A. L. \& Halpern, D. 2002 Stokes-flow instability due to interfacial surfactant. Phys. Fluids 14 (7), 1-4.

Frenkel, A. L. \& Halpern, D. 2017 Surfactant and gravity dependent instability of two-layer Couette flows and its nonlinear saturation. J. Fluid Mech. 826, 158-204.

Frenkel, A. L., Halpern, D. \& Schweiger, A. J. 2019a Surfactant- and gravity-dependent instability of two-layer channel flows: linear theory covering all wavelengths. Part 1. 'Longwave' regimes. J. Fluid Mech. 863, 150-184.

Frenkel, A. L., Halpern, D. \& Schweiger, A. J. 2019b Surfactant- and gravity-dependent instability of two-layer channel flows: linear theory covering all wavelengths. Part 2. Midwave regimes. J. Fluid Mech. 863, 185-214.

Gallagher, C. T., Leighton, D. T. \& McCready, M. J. 1996 Experimental investigation of a two-layer shearing instability in a cylindrical Couette cell. Phys. Fluids 8 (9), 2385-2392.

Georgantaki, A., Vlachogiannis, M. \& Bontozoglou, V. 2016 Measurements of the stabilisation of liquid film flow by the soluble surfactant sodium dodecyl sulfate (sds). Int. J. Multiphas. Flow 86, 28-34. 
Gupta, L. \& WASAN, D. T. 1974 Surface shear viscosity and related properties of adsorbed surfactant films. Ind. Eng. Chem. Fundamen. 13 (1), 26-33.

Halpern, D. \& Frenkel, A. L. 2003 Destabilization of a creeping flow by interfacial surfactant: linear theory extended to all wavenumbers. J. Fluid Mech. 485, 191-220.

Hooper, A. P. 1985 Long-wave instability at the interface between two viscous fluids: Thin layer effects. Phys. Fluids 28 (6), 1613-1618.

Hooper, A. P. \& Grimshaw, R. 1985 Nonlinear instability at the interface between two viscous fluids. Phys. Fluids 28 (1), 37-45.

Jensen, O. E. \& Grotberg, J. B. 1993 The spreading of heat or soluble surfactant along a thin liquid film. Phys. Fluids 5, 58-68.

KAlogirou, A. 2018 Instability of two-layer film flows due to the interacting effects of surfactants, inertia and gravity. Phys. Fluids 30 (030707), 1-12.

Kalogirou, A. \& Blyth, M. G. 2019 The role of soluble surfactants in the linear stability of two-layer flow in a channel. J. Fluid Mech. 873, 18-48.

Kalogirou, A. \& Papageorgiou, D. T. 2016 Nonlinear dynamics of surfactant-laden two-fluid Couette flows in the presence of inertia. J. Fluid Mech. 802, 5-36.

Kalogirou, A., Papageorgiou, D. T. \& Smyrlis, Y.-S. 2012 Surfactant destabilisation and nonlinear phenomena in two-fluid shear flows at small Reynolds numbers. IMA J. Appl. Math. 77, 351-360.

Langevin, D. 2014 Rheology of adsorbed surfactant monolayers at fluid surfaces. Annu. Rev. Fluid Mech. 46, 47-65.

Maisch, P., Eisenhofer, L. M., Tam, K. C., Distler, A., Voigt, M. M., Brabec, C. J. \& EGELHAAF, H.-J. 2019 A generic surfactant-free approach to overcome wetting limitations and its application to improve inkjet-printed P3HT:non-fullerene acceptor PV. J. Mater. Chem. A 7, 13215-13224.

Mavromoustaki, A., Matar, O. \& Craster, R. V. 2012a Dynamics of a climbing surfactantladen film - I: Base-state flow. J. Colloid Interf. Sci. 371, 107-120.

Mavromoustaki, A., Matar, O. \& Craster, R. V. 2012b Dynamics of a climbing surfactantladen film - II: Stability. J. Colloid Interf. Sci. 371, 121-135.

Morgan, C. E., Breward, C. J. W., Griffiths, I. M., Howell, P. D., Penfold, J., Thomas, R. K., Tucker, I., Petkov, J. T. \& Webster, J. R. P. 2012 Kinetics of surfactant desorption at an air-solution interface. Langmuir 28, 17339-17348.

Ooms, G., Segal, A. \& Cheung, S. Y. 1985 Propagation of long waves of finite amplitude at the interface of two viscous fluids. Int. J. Multiph. Flow 11, 481-502.

Orzag, S. A. 1971 Accurate solution of the Orr-Sommerfeld stability equation. J. Fluid Mech. 50, 659-703.

Pereira, A. \& Kalliadasis, S. 2008 Dynamics of a falling film with solutal Marangoni effect. Phys. Rev. E 78 (036312), 1-19.

Petkova, R., Tcholakova, S. \& Denkov, N. D. 2012 Foaming and foam stability for mixed polymer-surfactant solutions: Effects of surfactant type and polymer charge. Langmuir 28, 4996-5009.

Phan, C.M., NGuyen, A.V. \& Evans, G.M. 2005 Dynamic adsorption of sodium dodecylbenzene sulphonate and dowfroth 250 onto the air-water interface. Miner. Eng. 18, 599-603.

Picardo, J. R., Radhakrishna, T. G. \& Pushpavanam, S. 2016 Solutal Marangoni instability in layered two-phase flows. J. Fluid Mech. 793, 280-315.

Ponce-Torres, A., Montanero, J. M., Herrada, M. A., Vega, E. J. \& Vega, J. M. 2017 Influence of the surface viscosity on the breakup of a surfactant-laden drop. Phys. Rev. Lett. 118 (024501), 1-5.

Power, H., Villegas, M. \& Carmona, C. 1991 Nonlinear inertial effects on the instability of a single long wave of finite amplitude at the interface of two viscous fluids. J. Appl. Math. Phys. (ZAMP) 42, 663-679.

Pozrikidis, C. 2004 Instability of multi-layer channel and film flows. Adv. Appl. Mech. 40, $179-239$.

RENARDY, Y. 1989 Weakly nonlinear behavior of periodic disturbances in two-layer CouettePoiseuille flow. Phys. Fluids A 1 (10), 1666-1676. 
RENARDY, Y. Y. 1985 Instability at the interface between two shearing fluids in a channel. Phys. Fluids 28 (12), 3441-3443.

Samanta, A. 2013 Effect of surfactant on two-layer channel flow. J. Fluid Mech. 735, 519-552.

Shen, A. Q., Gleason, B., McKinley, G. H. \& Stone, H. A. 2002 Fiber coating with surfactant solutions. Phys. Fluids 14, 4055-4068.

Song, Q., Couzis, A., Somasundaran, P. \& Maldarelli, C. 2006 A transport model for the adsorption of surfactant from micelle solutions onto a clean air/water interface in the limit of rapid aggregate disassembly relative to diffusion and supporting dynamic tension experiments. Colloids Surface A 282-283, 162-182.

Thompson, J. \& Blyth, M. G. 2016 Inertialess multilayer film flow with surfactant: Stability and traveling waves. Phys. Rev. Fluids 1 (063904), 1-41.

Tilley, B. S., Davis, S. H. \& Bankoff, S. G. 1994a Linear stability theory of two-layer fluid flow in an inclined channel. Phys. Fluids 6 (12), 3906-3922.

Tilley, B. S., Davis, S. H. \& Bankoff, S. G. 1994b Nonlinear long-wave stability of superposed fluids in an inclined channel. J. Fluid Mech. 277, 55-83.

Trefethen, L. N. 2000 Spectral Methods in Matlab. Philadelphia: Society for Industrial and Applied Mathematics.

WEI, H.-H. 2005 On the flow-induced Marangoni instability due to the presence of surfactant. J. Fluid Mech. 544, 173-200.

Weinstein, S. J. \& Ruschak, K. J. 2004 Coating flows. Annu. Rev. Fluid Mech. 36, 29-53.

Yıн, C.-H. 1967 Instability due to viscosity stratification. J. Fluid Mech. 27, 337-352.

Zell, Z. A., Nowbahar, A., Mansard, V., Leal, L. G., Deshmukh, S. S., Mecca, J. M., Tucker, C. J. \& Squires, T. M. 2014 Surface shear inviscidity of soluble surfactants. Proc. Natl. Acad. Sci. USA 111 (10), 3677-3682. 